\title{
Vascular tropism models of blood-borne microbial dissemination
}

\author{
Anna E. Boczula ${ }^{1}$, Amy Ly ${ }^{1}$, Rhodaba Ebady ${ }^{1}$, Janet Cho $^{1}$, Zoha Anjum ${ }^{1}$, Nataliya Zlotnikov ${ }^{1}$, \\ Henrik Persson ${ }^{2}$, Tanya Odisho ${ }^{1}$, Craig A. Simmons ${ }^{2}$ and Tara J. Moriarty ${ }^{1 *}$
}

${ }^{*}$ Corresponding author. ${ }^{1}$ Faculties of Dentistry and Medicine (Department of Laboratory Medicine and Pathobiology), University of Toronto. 124 Edward Street Rm 555. Toronto ON, Canada M5G 1G6 tara.moriarty@utoronto.ca. Phone: 416-978-6685.

Word count: Introductory summary (255), Main text, excluding table, legends, methods (2652) References: Main text, excluding Methods \& Supplemental Materials (50)

Key words: bloodborne pathogen, dissemination, Lyme disease, tropism, endothelium

Competing interests. The authors declare that the research was conducted in the absence of any commercial or financial relationships that could be construed as a potential conflict of interest The authors declare no competing interests.

Acknowledgements. Critical review of manuscript: Moriarty lab members. Technical support: Peter Gilgan Centre for Research Live Cell Imaging Facility; University of Toronto Division of Comparative Medicine.

Funding Information. Canadian Institutes of Health Research (CIHR) (MOP-119598, CPG127788, PJT-159466-S); Natural Sciences and Engineering Research Council (NSERC) (RGPIN 401938-11, RGPIN-2017-06403, CHRPJ 446650-13); Canada Foundation for Innovation (27881, IOF 27881). Scholarships: Queen Elizabeth II Graduate Scholarship in Science and Technology (AB), Harron Fellowship (RE), Ontario Graduate Scholarship (RE), University of Toronto Graduate Fellowship (AB, AL, RE)

Author Contributions. Conceptualization (TJM, AB, CAS), data curation (AB, TJM, AL, JC ), formal analysis (AB, TJM, AL, JC, RE), funding acquisition (TJM, AB, CAS), investigation (AB, ZA, AL, RE, NZ, HP, TJM, TO, JC ), methodology (AB, TJM, RE), project administration (TJM), resources (TJM, CAS), software (TJM, AB), supervision (TJM, CAS), validation (TJM, AB), visualization ( $\mathrm{AB}, \mathrm{AL}, \mathrm{NZ}, \mathrm{RE})$, writing-original draft preparation (AB, TJM), writing- review and editing (TJM, AB, AL, JC). 


\section{SUMMARY}

2 Similar to circulating tumour and immune cells, many blood-borne microbes preferentially "home" to specific vascular sites and tissues during hematogenous dissemination ${ }^{1-5}$. For many pathogens, the "postal codes" and mechanisms responsible for tissue-specific vascular tropism are unknown and have been challenging to unravel. Members of the Lyme disease Borreliella burgdorferi species complex infect a broad range of mammalian tissues and exhibit complex strain-, species- and host-specific tissue tropism patterns. Intravenous perfusion experiments and intravital microscopy studies suggest that heterogeneous tissue tropism properties may depend on tissue-specific differences in host and microbial molecules supporting vascular interaction and extravasation. However, interpreting these studies can be complicated because of the immuneprotective moonlighting (multitasking) properties of many B. burgdorferi adhesins. Here, we investigated whether B. burgdorferi vascular interaction properties measured by live cell imaging and particle tracking in aorta, bladder, brain, joint and skin microvascular flow chamber models predict strain- and tissue-specific dissemination patterns in vivo These studies identified strainand endothelial cell type-specific interaction properties that accurately predicted in vivo dissemination of B. burgdorferi to bladder, brain, joint and skin but not aorta, and indicated that dissemination mechanisms in all of these tissues are distinct. Thus, the ability to interact with vascular surfaces under physiological shear stress is a key determinant of tissue-specific tropism for Lyme disease bacteria. The methods and model systems reported here will be invaluable for identifying and characterizing the diverse, largely undefined molecules and mechanisms supporting dissemination of Lyme disease bacteria. These methods and models may be useful for studying tissue tropism and vascular dissemination mechanisms of other blood-borne microbes.

\section{MAIN TEXT}

Generation of hTERT-immortalized primary human endothelial cells (ECs) from tissues endothelial cell (EC) interactions is the challenge of cultivating a sufficient number of primary human ECs, which undergo replicative senescence and phenotypic drift in culture. The first goal of our studies was to develop stable, replicable flow chamber models for studying bacterialendothelial interaction mechanisms. Immortalization of ECs without loss of characteristic 
1 Transcriptase (hTERT) ${ }^{6}$. We immortalized primary human ECs derived from umbilical vein 2 (HUVEC), aorta, bladder, brain and joint synovial microvessels by retroviral hTERT expression (Fig. 1; Fig. S1). We attempted to immortalize cardiac, dermal, liver and lung microvascular ECs, but found that these cell types did not grow well, quickly lost their parent morphology in culture, or could not be immortalized (data not shown). hTERT expression successfully immortalized HUVEC, aorta, bladder, brain and joint ECs, as determined by comparing population doubling (PD) times of parental and hTERT-expressing progeny after 9-12 weeks of cultivation (Fig. 1A, 8 Fig S1A).

To determine if immortalized ECs retained primary EC properties we measured intercellular junction expression, localization of the EC marker VE-cadherin (Fig. 1B, S1B), and endothelial surface glycocalyx thickness (Fig. 1C) ${ }^{7,8}$. Immortalization reduced VE-cadherin expression in aorta, bladder and joint ECs, but not HUVEC or brain ECs. (Fig. 1B, S1B). Glycocalyx thickness measured by live cell imaging under flow was similar in parent and immortalized progeny for all EC types except aorta (Fig. 1C), and was comparable to EC glycocalyx thickness measured by cryoelectron microscopy ${ }^{9}$.

Finally, we determined if immortalization affected $B$. burgdorferi-endothelial interactions in flow chambers under postcapillary venule shear stress conditions at which B. burgdorferivascular interactions have previously been observed $\left(1 \mathrm{dyn} / \mathrm{cm}^{2}\right)^{10-15}$. We used three genetically distinct infectious strains differing in carriage of plasmid 1p25 or the gene encoding vascular adhesin BBK32 (+lp25+bbk32, -lp25+bbk32, -lp25-bbk32) ${ }^{10,11}$. These strains exhibit distinct dermal postcapillary venule interaction properties in live mice which are recapitulated with HUVEC in flow chambers at $1 \mathrm{dyn} / \mathrm{cm}^{2} 10-15$. We counted interactions manually and using particletracking methods (Fig 1D) to capture total (bacteria moving $<175 \mu \mathrm{m} / \mathrm{sec}$ ) and slow (bacteria moving $<125 \mu \mathrm{m} / \mathrm{sec}$ ) interactions for each strain and EC type. Manual counts measure the number of B. burgdorferi moving at these velocities over EC surfaces, whereas particle tracking captures numbers of binding-unbinding adhesion events that occur as bacteria move over surfaces at these velocities; each bacterial interaction trajectory consists of series of binding-unbinding events ${ }^{13}$. particle tracking, for EC types where VE-cadherin expression or glycocalyx thickness were reduced by immortalization ( $\mathrm{p} \geq 0.42$; Fig. S1: aorta, bladder, joint) and for EC types where immortalization did not disrupt these parameters ( $\mathrm{p} \geq 0.19$; Fig. 1E: brain, HUVEC). Although 

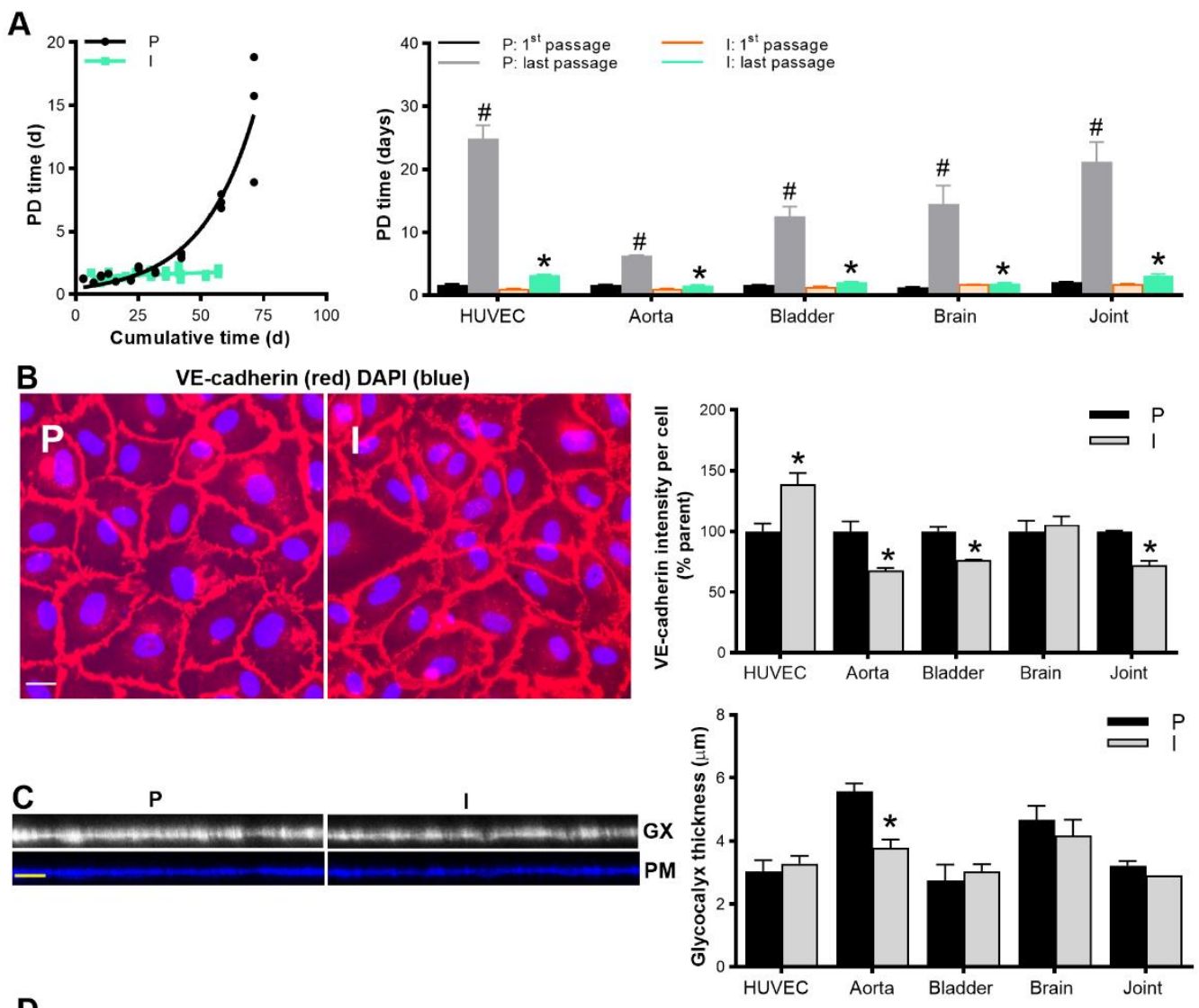

D

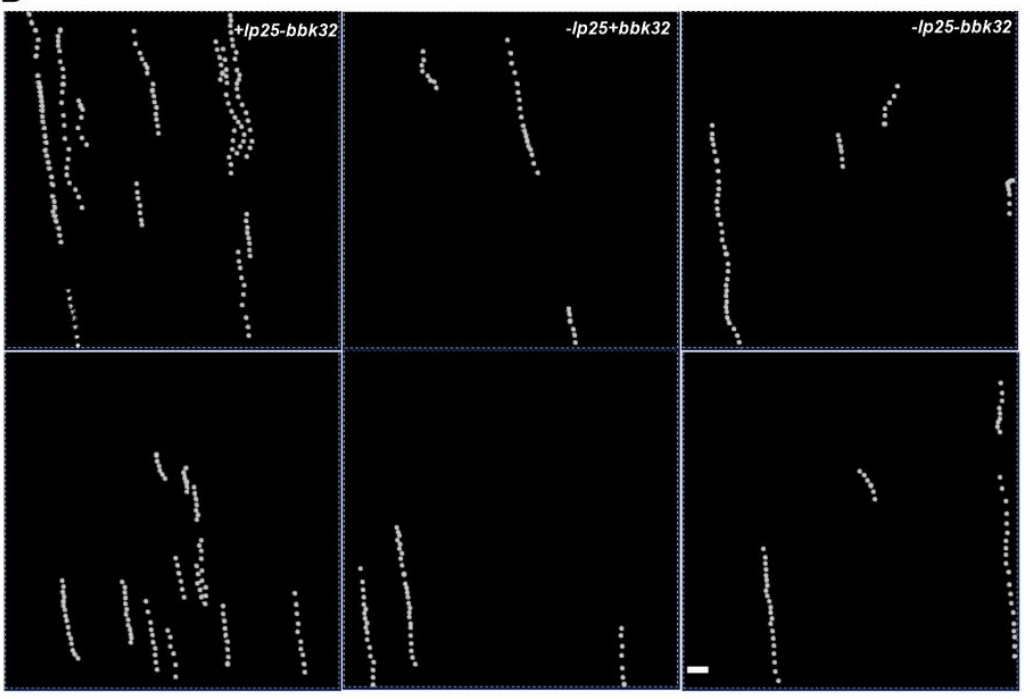

E
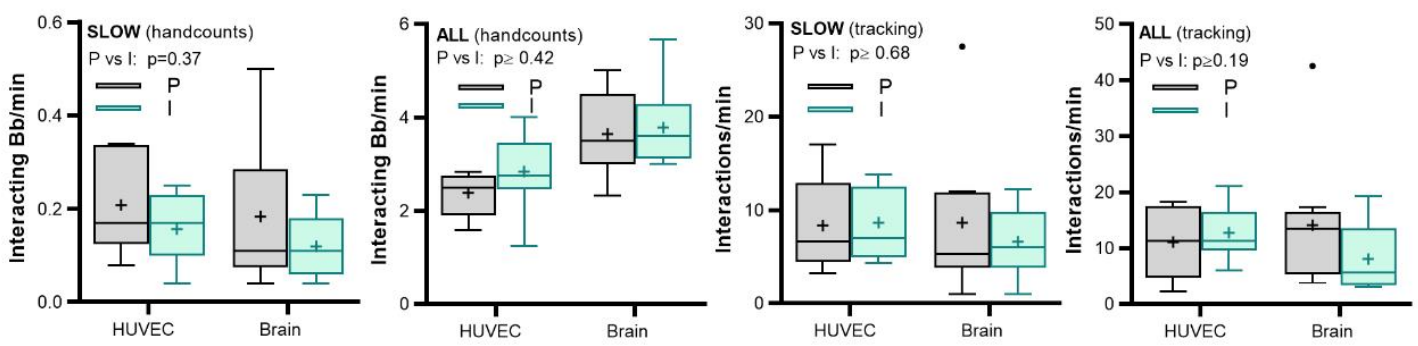
Fig 1: Generation and characterization of hTERT-immortalized primary human endothelial cells. Primary human endothelial cells (ECs) from vascular beds of indicated tissues immortalized by retrovirus-driven expression of human telomerase reverse transcriptase (hTERT). HUVEC are umbilical vein-derived ECs. (A) Sample non-linear regression showing change in population doubling time (PD) over time in culture for parent $(\mathrm{P})$ and immortalized (I) brain microvascular ECs (left). PD plots and immunofluorescence (IF) images for other ECs are shown in Fig. S1. Mean \pm SEM PD time at first and last passage for P and I ECs (right). (B) Representative epifluorescence IF photomicrographs of brain P and I ECs stained with antibody against endothelial marker VE-cadherin, and mean \pm SEM VE-cadherin intensity/cell for all ECs. (C) Representative confocal 3D projection images of live brain ECs visualized under flow with fluorescent plasma membrane (PM) dye and wheat germ agglutinin (GX: glycocalyx), and mean \pm SEM glycocalyx thickness for all ECs. (D) Sample 1 min time-lapse projection images of three genetically distinct bacterial strains (+lp25+bbk32, -lp25+bbk32, -lp25-bbk32) interacting with brain ECs under flow, captured by particle tracking. (E) Data are shown as Tukey box and whiskers plots of total (all) and slow (velocity $<125 \mu \mathrm{m} / \mathrm{s}$ ) interaction numbers under flow for all bacterial strains and HUVEC or brain ECs, measured by manual and particle tracking enumeration (hand counts, tracking, respectively). For all Fig. 1 experiments, $\mathrm{N} \geq 3$ independent EC and bacterial cultures. Statistics: two-way ANOVA of log-transformed values with Holm-Sidak post-tests. * indicates $\mathrm{p}<0.05 \mathrm{P}$ vs I (B, C, E, A: last passage). \# indicates $\mathrm{p}<0.051^{\text {st }}$ vs last passage for P or I ECs (A). Scale bars: 30 (B, C) and 20 (D) $\mu \mathrm{m}$.

immortalization did not affect interaction numbers for aorta, bladder or joint ECs, we conducted subsequent analyses using data only from parent ECs for these cell types to ensure that unanticipated artefacts were not introduced, whereas analyses for HUVEC and brain ECs were conducted with both parent and immortalized cells.

\section{Genetically distinct B. burgdorferi strains exhibit different tissue tropism patterns following} intravenous inoculation. Although it seems reasonable to assume that bacterial ability to interact with ECs under physiologically relevant fluid shear stress would affect their ability to disseminate out of the vasculature to specific tissues (tissue tropism), this hypothesis has not been formally tested. To test this hypothesis, we compared in vitro endothelial tropism patterns for different $B$. burgdorferi strains to in vivo tropism patterns in corresponding tissues measured in in vivo perfusion experiments (Fig. 2, S2). We used a short term intravenous (IV) perfusion model employed previously for tissue tropism and bloodstream survival studies ${ }^{16,17}$, but adapted this model to permit inter-strain and inter-mouse comparisons independent of potential inoculation and immune clearance differences among $\mathrm{C} 3 \mathrm{H} / \mathrm{HeN}$ mice and bacterial strains. In the original model, blood is collected $1 \mathrm{~h}$ after inoculation of mice, followed by intracardiac saline perfusion to remove unattached bacteria, and quantitative real-time PCR (qPCR) to measure bacterial burden. To control for potential clearance and inoculation differences among bacterial strains and mice, we expressed dissemination to each tissue in individual mice as a percentage of total dissemination to all tissues measured in the same animal. Tissues examined were bladder, brain, heart, patella and skin, reflecting the EC types used in flow chamber experiments. Studies were performed at one 
1 hour, one day and one week after inoculation in

2 both male and female mice to capture

3 dissemination to all tissues independent of

4 potential differences in dissemination kinetics,

5 and to determine if sex affected outcomes. effective model of $B$. burgdorferi murine dermal microvascular interactions ${ }^{13-15}$. The aorta and brain human ECs used in our studies were previously found to have similar characteristics as corresponding EC types in mice ${ }^{18-22}$. To the best of our knowledge, comparisons of primary bladder and joint human and mouse ECs have not been reported.

Unexpectedly, total burden across all tissues and at all timepoints was at least 1,000-fold greater in females than males (Fig. S2A). Although early strain specific dissemination patterns were similar in male and female mice, surprisingly we found that no strain disseminated appreciably or more than transiently to bladder or brain in female mice, whereas dissemination to heart was attenuated in male mice (Fig. S2B). Sex-specific $B$. burgdorferi tissue tropism patterns have not been systematically
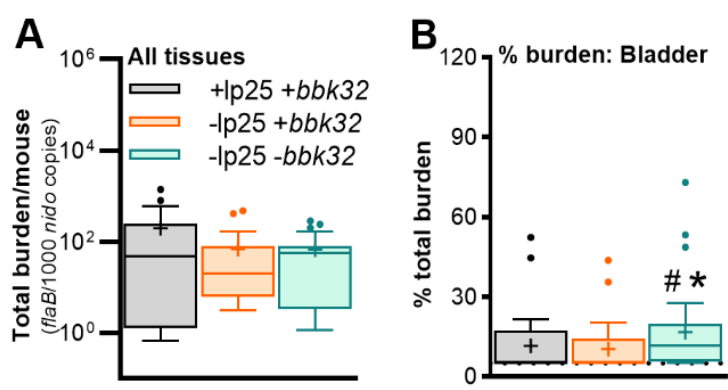

HUVEC has already been established as an
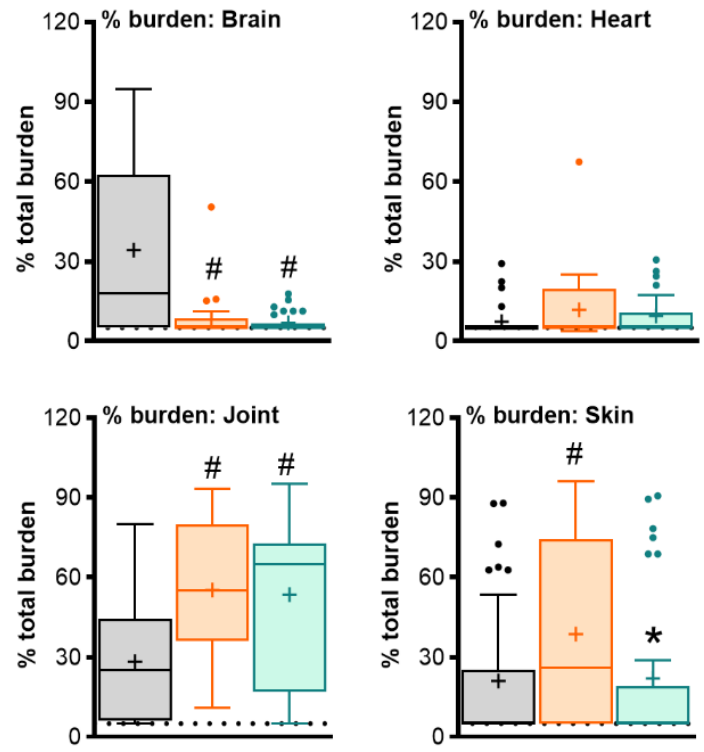

Fig 2: Genetically distinct $B$. burgdorferi strains exhibit different tissue tropism patterns following intravenous inoculation. Total B. burgdorferi burden in all tissues (A) and percent of total burden in indicated tissues after intravenous inoculation of male mice (B), measured by quantitative PCR. Shown are values for all timepoints $1 \mathrm{~h}, 1 \mathrm{~d}, 1 \mathrm{w}$. Values for individual timepoints for male and female mice are provided in Fig. S2. Data are depicted as Tukey box and whiskers plots. $\mathrm{N} \geq 28$ mice/experimental group. Statistics: Kruskal-Wallis ANOVA of log-transformed values, with Dunn's post-tests. * indicates $\mathrm{p}<0.05$ vs lp25-bbk32. \# indicates $\mathrm{p}<0.05 \mathrm{vs}+\mathrm{lp} 25+b b k 32$. investigated, although we have previously noted sex-specific differences in brain and heart burden in long-term mouse infections with the $-1 \mathrm{p} 25+b b k 32$ strain ${ }^{23}$. Since we could not be certain that the dissemination patterns in females were not artefacts due to extremely high overall burdens, we conducted subsequent analysis using dissemination data from male mice (Fig. 2).

Consistent with earlier studies implicating BBK32 in dermal vascular interactions and dissemination to skin ${ }^{10,12,13,24}$, BBK32-expressing strain -lp25+bbk32 disseminated to skin more 
efficiently than BBK32-deficient strain -lp25+bbk32 (Fig. 2B). Also as observed ${ }^{25}$, BBK32 was not required for joint dissemination (Fig. 2B). Surprisingly, BBK32 expression in an lp25-deficient background (-lp25+bbk32) was associated with impaired dissemination to bladder (Fig. 2B). As reported previously for dermal postcapillary venule interactions, lp25 carriage suppressed skin dissemination $^{10}$, but also suppressed dissemination to joint and promoted dissemination to brain (Fig. 2B). No-strain specific dissemination differences were observed in heart (Fig. 2B).

\section{Bacterial-endothelial interaction numbers in flow chambers predict dissemination patterns}

in vivo. We next investigated which, if any, interaction properties measured for a specific bacterial strain and human EC type in flow chambers were associated with/predictive of dissemination to the corresponding murine tissue in perfusion studies (Fig. 3, S1; Table 1). B. burgdorferi interacts with ECs under flow conditions through transient interactions called dragging and tethering (Fig. 3A), as well as by stationary adhesion ${ }^{11}$. Although stationary adhesion was once hypothesized to be the interaction step preceding B. burgdorferi extravasation, extravasation has only been directly observed following transient interactions ${ }^{11}$. We found that stationary adhesion on ECs in flow chambers did not correlate with dissemination to any tissue examined (Table 1), implying that stationary adhesion is likely not a major determinant of tissue-specific dissemination.

B. burgdorferi has a flat sine wave morphology and can interact with surfaces in flat or raised orientations (Fig. 3A) ${ }^{13,26}$. Dragging B. burgdorferi moves slowly over EC surfaces $(<125$ $\mu \mathrm{m} / \mathrm{s}$ ) under flow, with the edge of its sine wave shape oriented perpendicular to the EC surface, and forms adhesion complexes that are not stabilized by tethers (Fig. 3A top: $-\mathrm{T}){ }^{13,14}$. In this position, mechanical load is transferred from an adhesion complex at one "sine wave peak" to an adhesion complex on the next peak in the wave, and during each adhesion and load transfer event, the bacteria are displaced $2.83 \mu \mathrm{m}$, or the distance between sine wave peaks. These interactions depend on adhesion complexes that can sustain greater force on the load-bearing bond $\left(F_{\mathrm{b}}\right)$ without dissociating and exhibit a smaller dissociation rate $\left(\mathrm{K}_{\mathrm{off}}\right)$. By contrast, tethering bacteria are oriented flat against EC surfaces under flow, displace further than $2.83 \mu \mathrm{m}$ during each successive interaction, and move faster than $125 \mu \mathrm{m} / \mathrm{s}$ over EC surfaces (Fig. 3A bottom: $+\mathrm{T}$ ) ${ }^{13,14}$. Tethered interactions are stabilized by as yet unidentified tethers that absorb/reduce the force imposed on load-bearing adhesion complexes under flow. 


\section{A}

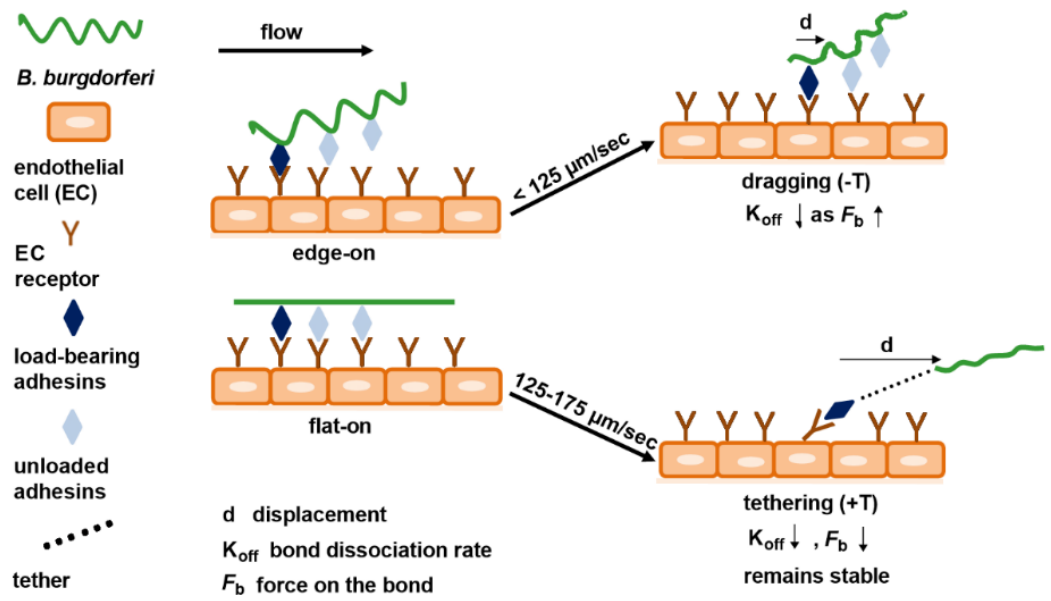

B
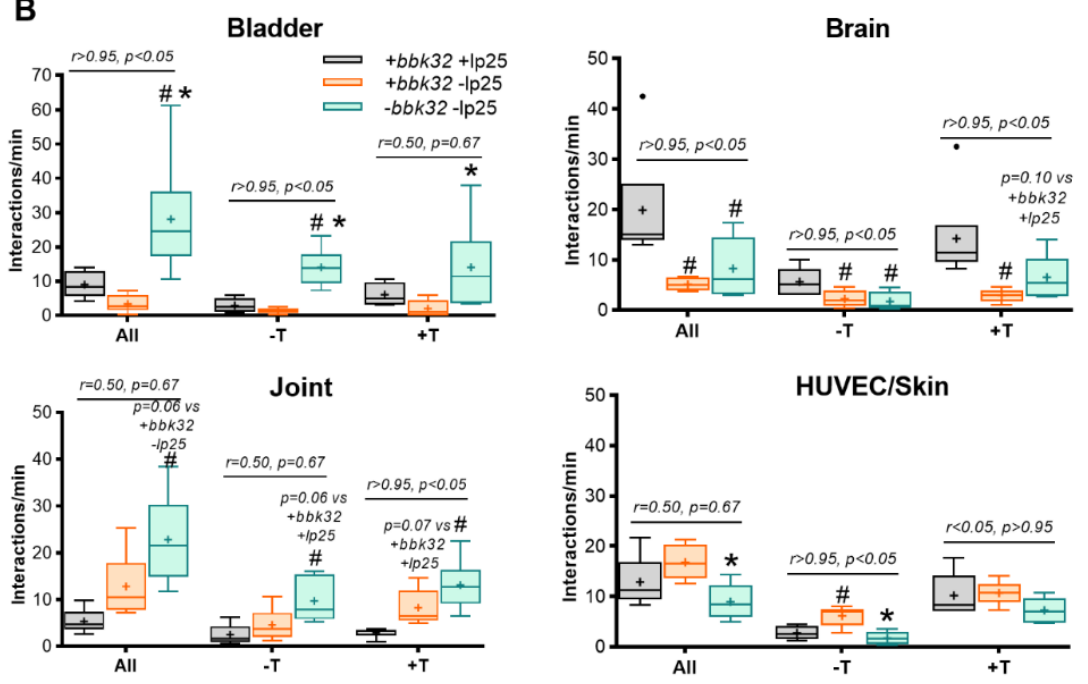

Fig. 3: Bacterial-endothelial interaction numbers in flow chambers predict dissemination patterns in vivo.

(A) Schematic depicting the two major modes of B. burgdorferi-EC interactions under physiological shear stress: Top: Slow, untethered (-T) "dragging" interactions in an edge-on orientation where mechanical load is transferred from peak to peak of the bacterial sine wave-shaped body (displacement, $\mathrm{d}$, is wavelength), and that dissociate more slowly $\left(\mathrm{K}_{\text {off }}\right)$ as force on the load-bearing adhesion bond $\left(F_{\mathrm{b}}\right)$ increases. Bottom: Faster interactions in a flat orientation stabilized by tethers $(+\mathrm{T})$ that slow dissociation by distributing mechanical load imposed on the adhesion bond; bacteria displace the length of the tether, which is further than peak-to-peak displacement in -T interactions. (B) $B$. burgdorferi-EC interaction number of interactions/min for all, $-\mathrm{T}$ (untethered) and $+\mathrm{T}$ (tethered) interactions in flow chambers, measured by particle tracking. Data shown as Tukey box and whiskers plots. $\mathrm{N} \geq 3$ biological replicates/experimental group. Statistical comparisons within each interaction group: ANOVA with Tukey's post-tests. $*$ indicates $\mathrm{p}<0.05$ vs $-1 \mathrm{p} 25+b b k 32$. \# indicates $\mathrm{p}<0.05 \mathrm{vs}+\mathrm{lp} 25+b b k 32$. Pearson correlation coefficients, $r$ (strain fold differences in tissue-specific burden in vivo vs strain fold differences in EC-specific flow chamber interaction numbers) and correlation $p$-values are indicated above each interaction group. Correlation values for all other interaction parameters are provided in Tables 1, S1, Fig. S3. 
To determine which, if any, interaction type or property predicted dissemination to corresponding tissues in vivo, we manually counted numbers of dragging and tethering bacteria and total interacting bacteria, and used particle tracking to identify and count total (Vel<175 All), tethering (Vel<175+T, Vel 125-175) and dragging interactions (Vel<125 All, Vel<175-T) (Table 1). We also investigated whether other biophysical interaction properties, including velocity, dissociation rate, displacement, force on the bond or number of successive interactions per trajectory were associated with tissue-specific in vivo dissemination patterns (Table S1; Fig. S3). To compare these variables, on widely different scales, to tissue burdens in vivo, we normalized the values for each parameter to the median value of the same parameter for the -lp25-bbk32 strain, then performed correlation analysis. Since B. burgdorferi-HUVEC interaction properties in flow chambers correspond to interaction properties measured in the skin of live mice by IVM ${ }^{13-15}$, we compared HUVEC interaction values to skin dissemination values in vivo. In vivo values used for comparison were pooled from all timepoints (Fig. 2). No significant correlations were observed for aorta ECs and heart, possibly because no significant strain differences were observed in hearts of male mice in vivo (Table 1) and possibly because mouse and human aorta ECs are subjected to much higher shear stress conditions in vivo $\left(>10 \mathrm{dyn} / \mathrm{cm}^{2}\right)^{27,28}$ than the shear stress used in our studies $\left(1 \mathrm{dyn} / \mathrm{cm}^{2}\right)$.

Table 1: Correlation analysis, tissue burden in vivo (male, 1h, 1d, 1w post IV injection) vs flow chamber interaction numbers

\begin{tabular}{|l|c|c|c|c|c|}
\hline Parameter $^{\boldsymbol{a}}$ & $\begin{array}{c}\text { Aorta/Heart } \\
(r, p)\end{array}$ & $\begin{array}{c}\text { Bladder } \\
(r, p)\end{array}$ & $\begin{array}{c}\text { Brain } \\
(r, p)\end{array}$ & $\begin{array}{c}\text { Joint } \\
(r, p)\end{array}$ & $\begin{array}{r}\text { HUVEC/Skin } \\
(r, p)\end{array}$ \\
\hline Dragging bacteria/min & $n / a^{\boldsymbol{c}}$ & $>\mathbf{0 . 9 5},<\mathbf{0 . 0 5}$ & $>\mathbf{0 . 9 5},<\mathbf{0 . 0 5}$ & $0.50,0.67$ & $>\mathbf{0 . 9 5},<\mathbf{0 . 0 5}$ \\
\hline Tethering bacteria/min & $n / a$ & $>\mathbf{0 . 9 5},<\mathbf{0 . 0 5}$ & $0.50,0.67$ & $>\mathbf{0 . 9 5},<\mathbf{0 . 0 5}$ & $0.50,0.67$ \\
\hline Total/min & $n / a$ & $>\mathbf{0 . 9 5},<\mathbf{0 . 0 5}$ & $0.50,0.67$ & $>\mathbf{0 . 9 5},<\mathbf{0 . 0 5}$ & $0.50,0.67$ \\
\hline Stationary adhesion/min & $n / a$ & $<0.05,>0.95$ & $-0.50,0.67$ & $<0.05,>0.95$ & $<0.05,>0.95$ \\
\hline Vel $<175 \mathrm{All}$ & $n / a$ & $>\mathbf{0 . 9 5},<\mathbf{0 . 0 5}$ & $>\mathbf{0 . 9 5},<\mathbf{0 . 0 5}$ & $0.50,0.67$ & $>\mathbf{0 . 9 5},<\mathbf{0 . 0 5}$ \\
\hline Vel<175-T & $n / a$ & $>\mathbf{0 . 9 5},<\mathbf{0 . 0 5}$ & $>\mathbf{0 . 9 5},<\mathbf{0 . 0 5}$ & $0.50,0.67$ & $>\mathbf{0 . 9 5},<\mathbf{0 . 0 5}$ \\
\hline Vel<175+T & $n / a$ & $0.50,0.67$ & $>0.95,0.18$ & $>\mathbf{0 . 9 5},<\mathbf{0 . 0 5}$ & $<0.05,>0.95$ \\
\hline Vel<125 All & $n / a$ & $>\mathbf{0 . 9 5},<\mathbf{0 . 0 5}$ & $>\mathbf{0 . 9 5},<\mathbf{0 . 0 5}$ & $0.50,0.67$ & $>\mathbf{0 . 9 5},<\mathbf{0 . 0 5}$ \\
\hline Vel 125-175 & $n / a$ & $0.50,0.67$ & $>\mathbf{0 . 9 5},<\mathbf{0 . 0 5}$ & $>\mathbf{0 . 9 5},<\mathbf{0 . 0 5}$ & $-0.50,0.67$ \\
\hline
\end{tabular}

${ }^{a}$ interaction parameters calculated from particle tracking data, organized by interaction type: all, $-T$ (untethered), $+T$ (tethered). ${ }^{b}$ Pearson correlation coefficients, $r$ (strain fold differences in tissue-specific burden in vivo vs strain fold differences in EC-specific flow chamber interaction numbers) and correlation p-values are indicated above each interaction group. ${ }^{c} n / a$ : values for all strains in heart in vivo are identical (male) were numbers of interactions measured by manual or particle tracking enumeration (Fig. 3B,

Table 1). Dissemination to bladder, brain and skin was associated most consistently with numbers 
1 of slow (velocity $<125 \mu \mathrm{m} / \mathrm{s}$ ), untethered (-T), dragging interactions observed on bladder and

2 HUVEC ECs in flow chambers, whereas joint dissemination was associated most strongly with 3 fast (velocity $>125 \mu \mathrm{m} / \mathrm{s}$ ), tethered (+T) interactions on joint ECs (Table 1). Dissemination 4 patterns in vivo were not generally correlated with interaction velocity, dissociation rate, 5 displacement, force on the bond or number of successive interactions per trajectory (Table 1, S1, Fig. S3), although it is possible that some of these properties might still be found to predict dissemination if flow chamber experiments are performed over a range of shear stress conditions that select for the most stable interactions ${ }^{13-15}$.

These observations implied that in bladder, brain, joint and skin, dissemination out of the blood stream was limited mainly by strain- and EC type-specific availability of bacterial adhesins and cognate EC receptors supporting transient, dynamic adhesive interactions. That is, the ratelimiting step for interactions is bond association rate. We conclude that EC interaction numbers in flow chambers robustly predict dissemination to corresponding tissues in vivo when tissue burden is adjusted for mouse- and bacterial strain-specific differences in overall burden, and that $B$. burgdorferi-vascular interactions are likely a rate-controlling step of dissemination in vivo.

Diverse tissue-specific vascular dissemination mechanisms. Consistent with previous sequencing studies of population bottlenecks during dissemination ${ }^{29}$, we found that all strains could interact with all ECs and disseminate to all tissues to some degree (Fig. 2, 3), implying that multiple dissemination mechanisms exist for most vascular beds and tissues. B. burgdorferi produces at least 19 surface-exposed adhesins, 17 of which have been identified as possible or confirmed mediators of tissue-specific dissemination and/or colonization, and roughly half of which are known or predicted to moonlight in immune evasion ${ }^{3,17,30-39}$. Strain- and speciesspecific polymorphisms in several B. burgdorferi adhesin genes also contribute to tissue-specific colonization patterns ${ }^{3,40}$. Thus, dissection of tissue-specific dissemination mechanisms in vivo can be challenging. Quantitative, easily genetically and biochemically manipulated in vitro models that eliminate confounding factors such as immune clearance and extravascular conditions affecting microbe proliferation will be helpful for characterizing these mechanisms. However, despite the existence of multiple dissemination mechanisms for each tissue, we found that interaction/dissemination patterns for each bacterial strain, EC type and corresponding tissue were 
different, implying that distinct primary vascular interaction mechanisms likely support 2 dissemination to each tissue (Fig. 2, 3B).

Bladder EC interactions and dissemination were 1p25-independent and suppressed by BBK32 (Fig. 2, 3B). Candidate mediators might include glycosaminoglycan- and laminin-binding Lmp1 and BB0406, which have been linked to bladder in intravenous phage display and long-term infectivity studies ${ }^{41-45}$. Since BBK32-expressing B. burgdorferi form a cap of polymerized plasma fibronectin at the mechanical load-bearing point of contact with ECs under flow ${ }^{14}$, it is possible that BBK32 suppresses bladder dissemination by directly or indirectly blocking access of bladderspecific adhesins to cognate bladder EC receptors.

Joint EC interactions and dissemination were BBK32-independent, as previously reported 16,25, and suppressed by lp25 (Fig. 2, 3B). Candidate joint mediators identified previously by intravenous phage display, intravital microscopy and infectivity studies include Lmp1, BB0406, BB0347, OspC and VlsE, as well as P66, an integral membrane protein that mediates joint vascular transmigration but not EC surface interactions $3,16,25,41,42,44,45$. These candidates bind a wide range of host molecules that could potentially be involved in joint vascular interactions and dissemination, including glycosaminoglycans, laminin, fibronectin, plasminogen and integrins. Of particular interest is $\mathrm{OspC}$, which supports transmigration out of the joint microvasculature in a fashion that depends on its extracellular matrix-binding properties ${ }^{3}$. It is not clear how or why joint EC interactions and dissemination would be suppressed by $1 \mathrm{p} 25$, which also suppresses HUVEC interactions and skin dissemination (Fig. 2, 3B). We examined tissue-specific colonization data from the genome-wide signature-tagged mutagenesis library generated by Lin and colleagues ${ }^{45}$, and found one lp25 locus (bbe27) where genetic disruption appeared to increase colonization of skin (ear) and joint compared to other tissues (bladder and heart) two weeks after infection. The bbe 27 open reading frames encodes conserved hypothetical protein of unknown function ${ }^{45}$ that does not appear to be membrane localized (data not shown). It will be interesting to determine if this locus can affect EC interactions and intravenous tissue dissemination.

As reported previously ${ }^{10,12,13}$, HUVEC interactions and skin dissemination were promoted by BBK32 and suppressed by lp25, but did not entirely depend on BBK32 (Fig. 2, 3B). Although B. garinii $\mathrm{DbpA} / \mathrm{B}$ has been found to support HUVEC interactions in flow chambers, $B$. burgdorferi $\mathrm{N} 40 \mathrm{DbpA} / \mathrm{B}$ does not ${ }^{46}$, and the potential contributions of the B. burgdorferi $\mathrm{B} 31$ $\mathrm{DbpA} / \mathrm{B}$ strain variant used in our studies to EC interactions and intravenous dissemination to skin 
have not been examined. Other candidates identified in intravenous infectivity studies are BB0406,

2 P66 and the fibronectin-binding adhesin RevA ${ }^{16,44}$.

Finally, we found that brain interactions and dissemination were BBK32-independent but promoted by lp25 (Fig. 2, 3B). Lp25 encodes the glycosaminoglycan-binding adhesin BptA, which is important for mouse infectivity in a tissue non-specific fashion (BptA-dependent brain colonization has not been examined) ${ }^{47}$. Lp25 carries one additional open reading frame (bbe09) identified as a locus important for mouse infectivity in genome-wide transposon mutagenesis studies ${ }^{45}$. The predicted gene product is a putative membrane-localized lipoprotein, P23, that is a member of the SP2/P23 conserved hypothetical protein family of unknown function ${ }^{45,48}$. Another member of this family, the gene product of unknown function encoded by the bbk52 locus on lp36, is transcriptionally upregulated in response to mammalian host conditions by the Rrp2, RpoN and RpoS regulators ${ }^{49}$, and is transcriptionally upregulated in the central nervous system of nonhuman primates infected with B. burgdorferi ${ }^{50}$. The potential contributions of BBE09, BBK52 and their encoding loci to B. burgdorferi pathogenesis have not yet been examined.

The ability to carefully dissect the molecular basis of tissue-specific vascular dissemination mechanisms using in vivo-validated model systems will be crucial for understanding the pathogenesis of $B$. burgdorferi and other blood-borne pathogens. These model systems also have strong potential for discovery of novel dissemination mechanisms using sequence-tagged random mutagenesis pathogen whole genome libraries and other, directed and undirected approaches.

\section{METHODS}

Ethics statement. This study was conducted in accordance with the most recent policies and Guide to the Care and Use of Experimental Animals by The Canadian Council on Animal Care. Animal work was approved by the University of Toronto Animal Care Committee in agreement with institutional guidelines (Protocols 20009347, 20009908 and 20010430). Work with Borrelia burgdorferi and primary human endothelial cells was approved by University of Toronto, Public Health Agency of Canada and Canadian Food Inspection Agency guidelines (University of Toronto biosafety permit 12a-M30-2).

Mouse strains and husbandry. Intravenous perfusion experiments were performed using 6- to 7week-old male C3H/HeN (Charles River Laboratories, Montréal, QC, Canada). Mice were housed in groups of 2 to 4 per environment-enriched cage with unlimited access to water and standard chow (Teklad 2018 Rodent Chow, Harlan Laboratories, Mississauga, ON, Canada). 
types used in experiments are described in Table S2. All primary human ECs were purchased from Lonza (Lonza Inc. Allendale, NJ, USA) or Cell Systems (Cell Systems, Kirkland, WA, USA) and cultured at $37{ }^{\circ} \mathrm{C}$ according to the manufacturer's instructions in tissue culture-treated T75 flasks without gelatin or Fn coating, in a humidified atmosphere containing $5 \% \mathrm{CO}_{2}$. Lonza cells were cultured in EGM-2 medium (Lonza cat. CC-3156) supplemented with bullet kits CC-4147 (all cells except HUVEC) or CC-4175 (HUVEC). Cell Systems cells were initially thawed and grown for the first three passages in CSC medium supplemented with Culture Boost (Cell Systems cat. CSS-A104) and 5\% heat-inactivated fetal bovine serum (FBS: Sigma-Aldrich Canada, Oakville, ON), then subsequently cultured in Lonza EGM-2 medium supplemented with bullet kit CC-4147. Cell Systems and Lonza cells were respectively frozen in Cell Freezing Medium (Cell Systems cat. 4Z0-705) or whole medium containing 1\% dimethyl sulfoxide (DMSO; Bioshop Canada, Burlington, ON) and 20\% heat-inactivated FBS. All ECs were passaged at $\sim 80 \%$ confluence. Lonza cells, rinsed with $37^{\circ} \mathrm{C}$ phosphate buffered saline without magnesium or calcium (PBS-/-: Multicell Wisent, St-Bruno, QC), trypsinized for 5 min at $37{ }^{\circ} \mathrm{C}$ with pre-warmed trypsin $(0.05 \%$ trypsin/0.53 mM EDTA; Life Technologies Canada, Burlington, ON), followed by neutralization with complete medium. Cell Systems cells were rinsed with PRG solution (EDTA -dPBS, Cell Systems cat. 4Z0-610), trypsinized for 0.5-2 min (until rounded up but not detached) with $37{ }^{\circ} \mathrm{C}$ PRG-2 solution (Trypsin/EDTA -dPBS Solution cat. 4Z0-310), followed by neutralization with an equal volume of ice-cold PRG-3 solution (Trypsin Inhibitor-dPBS Solution cat. 4Z0-410). After trypsinization, all cells were centrifuged at $220 \mathrm{xg}$ RT for $5 \mathrm{~min}$ followed by resuspension with fresh complete media and plated at 1:5 (Cell Systems cells)-1:10 (Lonza cells) dilutions. For all experiments only parent and immortalized ECs passaged fewer than eight times were used.

\section{Immortalization of primary human ECs.}

Retrovirus production. As previously described ${ }^{51}$, primary ECs were immortalized by infection with retrovirus encoding hTERT, produced by transfection of Gryphon amphotropic packaging cells (Allele Biotechnology, San Diego, CA, USA) with plasmid pBABE-hygrohTERT (cat. 1773; Addgene, Cambridge, MA, USA). Gryphon cells were cultivated according to manufacturer's instructions at $5 \% \mathrm{CO}_{2} 37^{\circ} \mathrm{C}$ in $10 \mathrm{~cm}$ tissue culture-treated dishes (VWR Canada, Mississauga, ON) in DMEM-high glucose (Life Technologies) supplemented with 10\% heat inactivated FBS and $100 \mathrm{U} / \mathrm{ml}$ penicillin/streptomycin (Thermo Fisher Scientific Canada, Burlington, ON). Gryphon cells were grown to $50-60 \%$ confluence by plating at 3.9-5.2 x $10^{6}$ cells $/ 10 \mathrm{~cm}$ plate $18-24 \mathrm{~h}$ before transfection. On the day of transfection, $12 \mu \mathrm{l}$ of FuGene HD transfection reagent (Promega, Madison, WI, USA) were added to RT serum-free Optimem medium (Fisher), followed by addition of $4 \mu \mathrm{g}$ pBABE-hygro-hTERT prepared by maxiprep (Qiagen Canada, Mississauga, ON), to a final volume of $200 \mu$ l. This mixture was incubated for $15 \mathrm{~min}$ at RT, then added to Gryphon cells, followed by incubation at $32{ }^{\circ} \mathrm{C} / 5 \% \mathrm{C}_{2}$ for $\sim 24 \mathrm{~h}$, when transfection medium was replaced with $10 \mathrm{ml}$ fresh complete growth medium. Retroviruscontaining supernatant was harvested at 48 and $72 \mathrm{~h}$ after medium change, centrifuged at $2000 \mathrm{xg}$ $4{ }^{\circ} \mathrm{C} 5 \mathrm{~min}$ to pellet cell debris, filter-sterilized with a $0.22 \mu \mathrm{m}$ filter and stored in $2 \mathrm{ml}$ single-use aliquots at $-80^{\circ} \mathrm{C}$.

Retroviral infection and polyclonal selection. On the day of retroviral infection of ECs, $4.6 \mathrm{ml}$ of $48 \mathrm{~h}$ viral supernatant mixed with $14.1 \mu \mathrm{l}$ of $8 \mu \mathrm{g} / \mathrm{ml}$ polybrene (Sigma) was added to 1.4-2.8 $\times 10^{6}$ ECs cultured to $20-30 \%$ confluence in a T75 flask, followed by $3 \mathrm{~h}$ incubation at $37{ }^{\circ} \mathrm{C}$ then replacement of virus-containing medium with complete growth medium. This infection was repeated $24 \mathrm{~h}$ later using the $72 \mathrm{~h}$ viral supernatant. To select for polyclonal populations in 
which hTERT-expressing retrovirus was stably integrated, $48 \mathrm{~h}$ after the last infection hygromycin B (BioBasic Canada, Markham, ON) was added to endothelial cells to $20 \mu \mathrm{g} / \mathrm{ml}$ and included in culture medium for all subsequent passages ${ }^{52}$. Hygromycin-resistant cells were frozen in whole medium containing $1 \%$ DMSO and $20 \%$ FBS upon reaching $80 \%$ confluence and at multiple passages thereafter.

Measurement of population doubling (PD) times. To determine if hygromycin-resistant cells were immortal, we measured PD times of triplicate cultures of parent and hTERT-expressing cells at each passage until $\sim 2$ weeks after parent cells reached replicative senescence ( 2-3 months total passaging time) ${ }^{52}$. Cells were counted at every passage (at 75-80\% confluence) using a Beckman coulter Z1 particle counter (Beckman Coulter Canada, Mississauga, ON), blood cell counter vials (VWR) and $500 \mu \mathrm{l}$ of a $10 \mathrm{ml}$ resuspension of cells from each flask diluted with 19.5 ml Isoton II Diluent (Beckman). PD time, in hours, was calculated from the formula PD Time $(\mathrm{PDT})=\mathrm{T}^{*} \ln 2 / \ln (\mathrm{Xe} / \mathrm{Xb})$, where $\mathrm{T}$ is incubation time in hours. $\mathrm{Xb}$ and $\mathrm{Xe}$ are cell number at the beginning and end of incubation time, respectively. PD times were converted to days and plotted against cumulative days of cultivation using non-weighted least squares regression and exponential growth equations fit in GraphPad Prism v. 8.4.3 (GraphPad Software, San Diego, CA, USA).

Visualization and quantification of VE-cadherin-labelled EC junctions. ECs grown in triplicate to two days post-confluence in 48-well/9.8 $\mathrm{mm}$ plates (Falcon, Fisher) were rinsed with $37{ }^{\circ} \mathrm{C}$ PBS containing calcium and magnesium (PBS+/+: Sigma), fixed in $-20{ }^{\circ} \mathrm{C}$ methanol at $-20{ }^{\circ} \mathrm{C}$ for $15 \mathrm{~min}$, rinsed with PBS -/- (Fisher), blocked for $20 \mathrm{~min}$ at $37^{\circ} \mathrm{C}$ in PBS-/- with $3 \%$ w/v bovine serum albumin (BSA) (Sigma), incubated at $37{ }^{\circ} \mathrm{C}$ for $1 \mathrm{~h}$ with a 1:333 dilution of 1 $\mathrm{mg} / \mathrm{ml}$ anti-VE-cadherin polyclonal antibody (Abcam, Toronto, ON, Canada, cat. ab33168) in PBS-/- with 3\% BSA, washed once with PBS-/-, blocked for $30 \mathrm{~min}$ at RT with $10 \%$ heatinactivated goat serum (Sigma) in PBS-/-, incubated in the dark for $1 \mathrm{~h}$ at RT with $5 \mu \mathrm{g} / \mathrm{ml}$ Alexa Fluor 488-conjugated goat anti-rabbit IgG $(\mathrm{H}+\mathrm{L})$ secondary antibody (Fisher) in PBS-/- with $10 \%$ goat serum, then washed three times with PBS-/-, followed by staining of cell nuclei for 3 min at RT with $1 \mu \mathrm{g} / \mathrm{ml}$ Hoechst 33342 (Sigma) in PBS-/-, and three final rinses with PBS-/-. Samples were stored in the dark at $4{ }^{\circ} \mathrm{C}$ until imaging.

Images were captured using an Olympus IX71 (Olympus, Tokyo, Japan) epifluorescence microscope equipped with a Retiga 2000R Fast-1394 camera (QImaging, Surrey, BC, Canada), XCite 120 series illumination source (Excelitas, Waltham, MA, US ), DAPI and TXRed filters (Semrock Inc., Rochester, NY,US), a 40X (NA 0.4) air objective (Olympus, Tokyo, Japan) and and a custom acquisition script based on ImageJ (LOCI, University of Wisconsin, USA) ${ }^{53}$ and Micro-Manager (Vale Lab, University of California, San Francisco, CA, US) ${ }^{54}$ software. Image acquisition conditions were identical for all samples. Exposure times were 100 and $2000 \mathrm{~ms}$ for DAPI and TXRed, respectively, with no frame averaging, 0.75X zoom, 32 bits/pixel (1200x 1600 pixels/image, 5.53 pixels/ $\mu \mathrm{m}$ ) and $0.18 \mu \mathrm{m}^{2} \mathrm{xy}$ pixel resolution.

Exported DAPI and TXRed 32-bit tiffs were converted to 8-bit in ImageJ, for all ECs, TXRed signal range was set to 200-800, and DAPI signal range was set to 200-800 (brain, HUVEC), 200-600 (aorta), 161-430 (bladder), 250-384 (joint).VE- cadherin signal intensity was measured in the TXReD images by subtraction of average background intensity (darkest $10 \times 10 \mu \mathrm{m}^{2}$ region of interest, ROI) from the average intensity measured in each of three $200 \times 200$ $\mu \mathrm{m}^{2}$ ROIs at three distinct locations in each image. The average of background-corrected signal intensity was measured per cell for the three ROIs in each image, and was expressed as a percent of the average intensity for parental EC images. 
Visualization and quantification of EC glycocalyx thickness. As previously described ${ }^{13}, \sim 1.6$ x $10^{5} \mathrm{ECs} / \mathrm{channel}$ were seeded in ibiTreat hydrophilic tissue-culture treated Ibidi $\mu$-Slides $\mathrm{VI}^{0.4}$ (Ibidi, Planegg, Martinsried, Germany) and cultivated to $2 \mathrm{~d}$ post-confluence with daily feeding (a total of $\sim 3 \mathrm{~d}$ ). ECs were labelled for $10 \mathrm{~min}$ at $37^{\circ} \mathrm{C}$ with $100 \mu \mathrm{l}$ of a 1:10,000 dilution of Alexa 647-wheat germ agglutinin (lectin) (Life Technologies) to label the glycocalyx (GX) and for $3 \mathrm{~min}$ with CellMask Green plasma membrane live cell imaging dye (Life Technologies) prepared in endothelial growth medium to label ECs, then rinsed with perfusion medium before imaging. Perfusion media containing Hank's Balanced Salt Solution (HBSS: Life Technologies), and $10 \%$ heat-inactivated FBS (Sigma) was loaded in $60 \mathrm{ml}$ syringes and perfused over endothelial monolayers in Ibidi chambers at $1.0 \mathrm{dyn} / \mathrm{cm}^{2}(34.1 \mathrm{ml} / \mathrm{h}$ ) using a syringe pump (Model: NE1000, New Era Pump Systems Inc., Farmingdale, NY, USA). Ambient temperature on the microscope stage was maintained at $28^{\circ} \mathrm{C}$ using an infrared heat lamp.

Z-series micrographs (512x512 pixels, zoom 1.25X, xy pixel size $0.73 \mu \mathrm{m}$, frame average 10 , line average 3 ) were collected simultaneously every $0.75 \mu \mathrm{m}$ in bidirectional resonant mode (pinhole 2.5 AU), at 511-600 nm (488 nm Argon laser, 10\% intensity, HyD detector BrightR mode, gain 100), and 664-721 nm (633 nm laser, 100\% intensity, HyD detector standard mode, gain 100), using an upright SP8 tandem scanner spectral confocal microscope equipped with a 25X 0.95 NA long working range water-immersion objective and operated by LAS X v.3.1.5.16308 software (Leica, Mannheim, Germany).

Glycocalyx thickness was measured using the line tool in Volocity v.6.5 (Quorum Technologies Inc., Puslinch, ON, Canada) at each of nine positions for each micrograph (xy pixel coordinates: $128 \times 128 ; 128 \times 256 ; 128 \times 384 ; 256 \times 128 ; 256 \times 256 ; 256 \times 384 ; 384 \times 128 ; 384 \times 256$; $384 \times 384$ ), with a minimum of five independent biological replicates/experimental group. As described, ${ }^{55}$ we first identified the positions of maximum intensity in each channel on the apical surface of endothelial cells. We calculated values for $25 \%$ maximum intensity, then, moving apically, identified the z-position at which the intensity in each channel reached $25 \%$ of the channel maximum. Glycocalyx thickness was calculated by subtracting the $25 \%$ intensity z-position in the plasma membrane channel ("outer edge" of plasma membrane) from the $25 \%$ intensity z-position in the glycocalyx channel ("outer edge" of glycocalyx). The median value from all nine measurements from the micrograph for each biological replicate $(n \geq 5)$ was used for subsequent calculations and statistical analysis.

Preparation of endothelial monolayers for flow chamber experiments. ECs cultivated and seeded on Ibidi devices as described above were labelled for $5 \mathrm{~min}$ at $37{ }^{\circ} \mathrm{C}$ with $100 \mu \mathrm{l}$ of a 1:2,000 dilution of CellMask Orange plasma membrane live cell imaging dye (Life Technologies) in endothelial growth medium, then rinsed with perfusion medium before imaging.

B. burgdorferi strains and preparation of bacteria for flow chamber experiments. All details of B. burgdorferi strains used in this study are described previously ${ }^{10-12}$ and in Table S4. As reported ${ }^{13}$ B. burgdorferi was grown, washed, resuspended to $4 \times 10^{8} / \mathrm{ml}$ in ice cold PBS-/-, declumped, then diluted to $1 \times 10^{8} / \mathrm{ml}$ in RT HBSS containing $10 \%$ heat-inactivated FBS immediately before imaging, except that bacteria were cultivated without addition of blood.

Live time lapse imaging conditions. Bacteria were perfused over endothelial monolayers in flow chambers as described in glycocalyx imaging section. For each biological replicate (independent endothelial and bacterial cultures) four 1-min xyt series $(512 \times 512$, zoom 1.50X, xy pixel size 
$0.607 \mu \mathrm{m}$, line average 2) were acquired simultaneously in bidirectional resonant mode (pinhole 10.74 AU, frames per sec 14.08) at 497-535 nm (488 nm Argon laser, 90-98\% intensity, HyD detector standard mode, gain 10) and 573-790 nm (561 nm laser, 9.8\% intensity, PMT detector, gain 450-500) using the Leica upright SP8 tandem scanner spectral confocal microscope equipped with a 25X 0.95 NA long working range water-immersion objective. Time lapse videos were independently inspected by at least two individuals to confirm monolayer confluence and that at least $75 \%$ of the endothelial surface was at the imaging focal plane for at least $75 \%$ of video duration. Videos that did not meet these criteria were excluded from subsequent analysis. Subsequent quality control and analysis steps in the time lapse bioinformatics pipeline are summarized below and in Fig. S4.

Manual counting of $\boldsymbol{B}$. burgdorferi-endothelial interactions under flow. Tethering (bacteria that pause but move faster than $125 \mu \mathrm{m} / \mathrm{s}$ ) and dragging (bacteria that move $<125 \mu \mathrm{m} / \mathrm{s}$ ) interactions and stationary adhesions (bacteria that stay at the same position for $\geq 20 \mathrm{~s}$ ) were manually enumerated in time lapse videos as described ${ }^{13}$, except that dragging was counted in three 30x $100 \mu \mathrm{m}$ ROIs positioned at the centre of the field of view, and the average of this value was used for subsequent analyses. Analysis to identify high certainty outlier interaction values in each experimental group (typically 12-24 videos) was performed in GraphPad Prism (ROUT Q $0.1 \%$ ). If outliers were identified in any video, it was removed from all subsequent analysis (Fig. S4). Interaction values for each biological replicate (independent bacterial and endothelial cultures) were calculated as the average of interaction values for all technical replicates (typically four videos). All subsequent statistical analysis was performed with values for biological replicates.

\section{Particle tracking and measuring physical properties of $B$. burgdorferi-endothelial} interactions under flow. Leica .lif library files were imported into Volocity, acquisition frame rates obtained from .lif files were entered manually, and the particle tracking was performed as reported ${ }^{13}$, with the following modifications (bioinformatics pipeline summarized in Fig. S4). Each video was analyzed using six tracking protocols (Table S4), and interactions were identified in the resulting trajectories using a previously described formula spreadsheet that identifies individual interactions in trajectories (decelerating bacteria) and calculates physical properties of these interactions (see Table S1 for parameters). To reduce potential user error in handling hundreds of thousands of lines of data from nearly 700 videos of data, macros were developed to permit batch data import into this formula spreadsheet without copying and pasting (Fig. S4). All parameter calculations and identification of untethered $(-\mathrm{T})$ and tethered $(+\mathrm{T})$ interactions were performed as reported ${ }^{13}$, except that for $K_{\text {off }}$ calculations time durations for interaction populations were binned in intervals of $0.07 \mathrm{~s}$, and linear regressions and runs tests were performed in GraphPad Prism using only interactions with durations $\leq 0.24 \mathrm{~s}$ ( $>95 \%$ of interactions). $\mathrm{R}^{2}$ values for all curves were $\geq 0.98$ and deviation from linearity was not significant $(\mathrm{p}>0.05)$.

Deduplication (arising from identification of the same trajectory with different tracking protocols) was performed by identifying interactions captured at the same time point in each video and examining their centroid xy position in the field of view. Interactions in the same quadrant were flagged as potential duplicates and manually verified in the original videos. Duplicate flagging using centroid position alone successfully identified duplicates in $>95 \%$ of cases (verified by manual inspection). When duplicates were identified, the interaction where measured bacterial length was longest was retained for subsequent analyses, and all other interactions were removed 
(Fig. S4). After deduplication, interactions were then filtered to eliminate non-specific interactions with displacement $\geq 45 \mu \mathrm{m}$, bacterial length $<2.5 \mu \mathrm{m}, \mathrm{F}_{b}$ (force on the bond) $<0.113 \mathrm{pN}$, velocity $>175 \mu \mathrm{m} / \mathrm{s}$, and trajectories with fewer than three successive interactions/track (Fig. S4).

\section{Intravenous (IV) perfusion experiments.}

Intravenous infection and perfusion. These experiments were performed largely as described by Caine et al. ${ }^{16}$ Briefly, ten mice per experimental group (90 total across 9 groups) were inoculated via tail vein with $1 \times 10^{8}$ bacteria washed twice in ice-cold PBS-/-, resuspended to $1 \times 10^{9} / \mathrm{ml}$ in PBS and injected using a 29-gauge needle (BD, Mississauga, ON, Canada) after warming to RT. At $1 \mathrm{~h}, 1$ day ( $24 \mathrm{~h}$ ) or 1 week, mice were anesthetized with $1 \%$ isoflurane or 100 mg ketamine/kg (Rogar/STB) of body weight and $10 \mathrm{mg} / \mathrm{kg}$ xylazine (MTC Pharmaceuticals), delivered by intraperitoneal injection. Anesthesia was confirmed by toe pinch, the heart was exposed, and $100 \mu \mathrm{l}$ of blood was collected using a small cut in the right atrium. A 24Gx.75in catheter (BD) was inserted into the left ventricle and mice were perfused with $20 \mathrm{ml} 0.9 \%$ sterile sodium chloride $(\mathrm{NaCl})$ at $4 \mathrm{ml} / \mathrm{min}$. Successful perfusion was confirmed by blanching of tissues. Whole bladder, ventral abdominal skin sections and right hemi-sections of heart, brain and patella were rinsed with $1 \mathrm{x}$ PBS-/-, blotted dry on tissue, snap-frozen on dry ice, then stored at $-80{ }^{\circ} \mathrm{C}$.

DNA extraction and qPCR measurement of bacterial and mouse DNA copy number. Total genomic DNA was isolated from tissues using a PureLink Genomic DNA Mini Kit (Fisher) and collected in $100 \mu \mathrm{l}$ kit elution buffer. Samples for which extraction was unsuccessful were excluded from further analysis. These were identified as samples with DNA concentration values $<10 \%$ of the median DNA concentration for the same tissue from the same experimental group $(<0.1 \%$ of samples). qPCR amplification of flaB and nido sequences was performed in technical sextuplicate and triplicate, respectively, as described ${ }^{56,57}$, except nido PCR conditions were: Step 1: $95^{\circ} \mathrm{C} 5 \mathrm{~min}$; Step 2: 50 cycles $98^{\circ} \mathrm{C} 6 \mathrm{~s}, 60{ }^{\circ} \mathrm{C} 3 \mathrm{~s}, 68{ }^{\circ} \mathrm{C} 5 \mathrm{~s}$; Step 3 melt curve analysis from $60-95^{\circ} \mathrm{C}$. Measurements with abnormal amplification curves, melting temperature or cycle threshold values more than 1.5 standard deviations from the mean for each biological replicate were excluded from subsequent analysis. Median $f l a B$ and nido copy number values for each sample were calculated from remaining values and standard curve values obtained for serial dilutions of known quantities of plasmids bearing flaB and nido sequences.

Clearance-adjusted measurement of tissue-specific dissemination. To control for potential effects of differences in bacterial clearance among experimental groups, differences in tissue cellularity and resulting nido values among tissues, and differences in the lowest detectable limit for flaB:nido ratios for tissues with distinct host cell densities, we calculated the median nido value for the same tissue from all experimental groups from all experiments, and multiplied the reciprocal of this value by the smallest number of flaB copies detectable by qPCR $(0.17$, or 1 copy detected per six technical replicates) to obtain the zero threshold flaB:nido for each tissue. To normalize the zero threshold for all tissues to the same value and adjust all flaB:nido values for tissue-specific differences in cell density, we calculated the zero threshold value for each tissue relative to the tissue with the lowest cell density (blood) then multiplied the flaB:nido values for samples from each tissue by the normalization value for that tissue compared to blood. All flaB:nido ratios for all tissues less than the universal zero threshold value (the threshold value for blood) were converted to the blood zero threshold value. 
Non-zero flaB:nido ratios that were 5-fold greater or less than $20 \%$ of the median value for the same experimental group and tissue were respectively converted to 5-fold maxima and $20 \%$ minima calculated using the median value for that group and tissue. We then calculated the sum of the flaB:nido ratios for all tissues in each mouse (referred to as "All" in Fig. 2A), identified mice with suboptimal tail vein injections ( $<10 \%$ of the median of sums of flaB:nido ratios in all tissues for each mouse in the same experimental group; <1\% of all mice in all experiments), and excluded these mice from subsequent analyses.

Finally, the percentage of total bacterial burden for each mouse localized to each tissue was calculated by comparing the tissue flaB:nido ratio to the sum of flaB:nido ratios for all tissues from the same mouse. To compare experimental group effect sizes to control groups, the percent total value for the same tissue from each mouse was expressed as a fold difference compared to the median percent total value for the same tissue in control mice.

\section{REFERENCES}

1. Margraf, A., Ley, K. \& Zarbock, A. Neutrophil recruitment: From model systems to tissuespecific patterns. Trends Immunol. 40, 613-634 (2019).

2. Jin, X. et al. A metastasis map of human cancer cell lines. Nature 588, 331-336 (2020).

3. Lin, Y.-P. et al. Strain-specific joint invasion and colonization by Lyme disease spirochetes is promoted by outer surface protein C. PLoS Pathog. 16, e1008516 (2020).

4. Lamond, N. M. et al. Cardiotropic isolates of Listeria monocytogenes with enhanced vertical transmission dependent upon the bacterial surface protein InlB. Infect Immun 89, e00321-20 (2021).

5. Bernabeu, M., Howard, C., Zheng, Y. \& Smith, J. D. Bioengineered 3D microvessels for investigating Plasmodium falciparum pathogenesis. Trends Parasitol S1471-4922, 30363-9 (2021).

6. Yang, J. et al. Human endothelial cell life extension by telomerase expression. J. Biol. Chem. 274, 26141-26148 (1999).

7. Zeng, Y., Zhang, X. F., Fu, B. M. \& Tarbell, J. M. The role of endothelial surface glycocalyx in mechanosensing and transduction. Adv. Exp. Med. Biol. 1097, 1-27 (2018).

8. Lampugnani, M. G., Dejana, E. \& Giampietro, C. Vascular endothelial (VE)-cadherin, endothelial adherens junctions, and vascular disease. Cold Spring Harb Perspect Biol 10, a029322 (2018).

9. Ebong, E. E., Macaluso, F. P., Spray, D. C. \& Tarbell, J. M. Imaging the endothelial glycocalyx in vitro by rapid freezing/freeze substitution transmission electron microscopy. Arterioscler. Thromb. Vasc. Biol. 31, 1908-1915 (2011).

10. Moriarty, T. J. et al. Vascular binding of a pathogen under shear force through mechanistically distinct sequential interactions with host macromolecules. Molecular Microbiology 86, 1116-1131 (2012).

11. Moriarty, T. J. et al. Real-time high resolution 3D imaging of the Lyme disease spirochete adhering to and escaping from the vasculature of a living host. PLoS Pathog. 4, e1000090 (2008).

12. Norman, M. U. et al. Molecular mechanisms involved in vascular interactions of the Lyme disease pathogen in a living host. PLoS Pathog. 4, e1000169 (2008). 
13. Ebady, R. et al. Biomechanics of Borrelia burgdorferi vascular interactions. Cell Rep 16, 2593-2604 (2016).

14. Niddam, A. F. et al. Plasma fibronectin stabilizes Borrelia burgdorferi-endothelial interactions under vascular shear stress by a catch-bond mechanism. Proc. Natl. Acad. Sci. U.S.A. 114, E3490-E3498 (2017).

15. Kao, W.-C. A. et al. Identification of Tp0751 (pallilysin) as a Treponema pallidum vascular adhesin by heterologous expression in the Lyme disease spirochete. Sci Rep 7, 1538 (2017).

16. Caine, J. A. \& Coburn, J. A short-term Borrelia burgdorferi infection model identifies tissue tropisms and bloodstream survival conferred by adhesion proteins. Infect. Immun. 83, 31843194 (2015).

17. Caine, J. A. et al. Borrelia burgdorferi outer surface protein $\mathrm{C}(\mathrm{OspC})$ binds complement component C4b and confers bloodstream survival. Cell. Microbiol. 19, e12786 (2017).

18. Shi, Y. et al. Publisher Correction: Rapid endothelial cytoskeletal reorganization enables early blood-brain barrier disruption and long-term ischaemic reperfusion brain injury. Nat Commun 11, 4335 (2020).

19. Niego, B. et al. Selective inhibition of brain endothelial Rho-kinase-2 provides optimal protection of an in vitro blood-brain barrier from tissue-type plasminogen activator and plasmin. PLoS One 12, e0177332 (2017).

20. Propson, N. E., Roy, E. R., Litvinchuk, A., Köhl, J. \& Zheng, H. Endothelial C3a receptor mediates vascular inflammation and blood-brain barrier permeability during aging. $J$ Clin Invest 131, e140966 (2021).

21. Yokoyama, M. et al. p53 plays a crucial role in endothelial dysfunction associated with hyperglycemia and ischemia. J Mol Cell Cardiol 129, 105-117 (2019).

22. Kho, J. et al. Argininosuccinate lyase deficiency causes an endothelial-dependent form of hypertension. Am J Hum Genet 103, 276-287 (2018).

23. Zlotnikov, N. et al. Infection with the Lyme disease pathogen suppresses innate immunity in mice with diet-induced obesity. Cell. Microbiol. 19, e12689 (2017).

24. Hyde, J. A. et al. Bioluminescent imaging of Borrelia burgdorferi in vivo demonstrates that the fibronectin-binding protein BBK32 is required for optimal infectivity. Mol Microbiol 82, 99-113 (2011).

25. Kumar, D. et al. Intravital imaging of vascular transmigration by the Lyme spirochete: Requirement for the integrin binding residues of the B. burgdorferi P66 protein. PLOS Pathog 11, e1005333 (2015).

26. Goldstein, S. F., Buttle, K. F. \& Charon, N. W. Structural analysis of the Leptospiraceae and Borrelia burgdorferi by high-voltage electron microscopy. J Bacteriol 178, 6539-45 (1996).

27. Gordon, E., Schimmel, L. \& Frye, M. The importance of mechanical forces for in vitro endothelial cell biology. Front Physiol 11, 684 (2020).

28. Feintuch, A. et al. Hemodynamics in the mouse aortic arch as assessed by MRI, ultrasound, and numerical modeling. Am J Physiol Heart Circ Physiol 292, H884-892 (2007).

29. Troy, E. B. et al. Understanding barriers to Borrelia burgdorferi dissemination during infection using massively parallel sequencing. Infect. Immun. 81, 2347-2357 (2013).

30. Caine, J. A. \& Coburn, J. Multifunctional and redundant roles of Borrelia burgdorferi outer surface proteins in tissue adhesion, colonization, and complement evasion. Front Immunol 7, 442 (2016).

31. Hyde, J. A. Borrelia burgdorferi keeps moving and carries on: a review of borrelial dissemination and invasion. Front Immunol 8, 114 (2017). 
32. Skare, J. T. \& Garcia, B. L. Complement evasion by Lyme disease spirochetes. Trends in Microbiology 28, 889-899 (2020).

33. Bernard, Q. et al. Borrelia burgdorferi protein interactions critical for microbial persistence in mammals. Cell Microbiol 21, e12885 (2019).

34. Curtis, M. W. et al. Characterization of stress and innate immunity resistance of wild-type and $\Delta$ p66 Borrelia burgdorferi. Infect. Immun. 86, e00186-17 (2018).

35. Pietikäinen, A. et al. Conserved lysine residues in decorin binding proteins of Borrelia garinii are critical in adhesion to human brain microvascular endothelial cells. Mol Microbiol Online ahead of print (2021) doi:10.1111/mmi.14687.

36. Tkáčová, Z. et al. Identification of the proteins of Borrelia garinii interacting with human brain microvascular endothelial cells. Ticks Tick Borne Dis 11, 101451 (2020).

37. Hahn, B. et al. BBB07 contributes to, but is not essential for, Borrelia burgdorferi infection in mice. Microbiology (Reading) 166, 988-994 (2020).

38. Saputra, E. P., Trzeciakowski, J. P. \& Hyde, J. A. Borrelia burgdorferi spatiotemporal regulation of transcriptional regulator bosR and decorin binding protein during murine infection. Sci Rep 10, 12534 (2020).

39. Schlachter, S., Seshu, J., Lin, T., Norris, S. \& Parveen, N. The Borrelia burgdorferi glycosaminoglycan binding protein Bgp in the B31 strain is not essential for infectivity despite facilitating adherence and tissue colonization. Infect. Immun. 86, e00667-17 (2018).

40. Tufts, D. M. et al. Outer surface protein polymorphisms linked to host-spirochete association in Lyme borreliae. Mol. Microbiol. 111, 868-882 (2019).

41. Antonara, S., Chafel, R. M., LaFrance, M. \& Coburn, J. Borrelia burgdorferi adhesins identified using in vivo phage display. Mol Microbiology 66, 262-276 (2007).

42. Yang, X., Coleman, A. S., Anguita, J. \& Pal, U. A chromosomally encoded virulence factor protects the Lyme disease pathogen against host-adaptive immunity. PLoS Pathog $\mathbf{5}$, e1000326 (2009).

43. Yang, X. et al. Middle region of the Borrelia burgdorferi surface-located protein 1 (Lmp1) interacts with host chondroitin-6-sulfate and independently facilitates infection. Cell. Microbiol. 18, 97-110 (2016).

44. Bista, S. et al. A novel laminin-binding protein mediates microbial-endothelial cell interactions and facilitates dissemination of Lyme disease pathogens. J Infect Dis 221, 14381447 (2020).

45. Lin, T. et al. Analysis of an ordered, comprehensive STM mutant library in infectious Borrelia burgdorferi: insights into the genes required for mouse infectivity. PLOS ONE 7, e47532 (2012).

46. Salo, J. et al. Flow-tolerant adhesion of a bacterial pathogen to human endothelial cells through interaction with biglycan. J. Infect. Dis. 213, 1623-1631 (2016).

47. Revel, A. T. et al. bptA (bbe16) is essential for the persistence of the Lyme disease spirochete, Borrelia burgdorferi, in its natural tick vector. Proceedings of the National Academy of Sciences of the United States of America 102, 6972-7 (2005).

48. Purser, J. E. \& Norris, S. J. Correlation between plasmid content and infectivity in Borrelia burgdorferi. Proc Natl Acad Sci U S A 97, 13865-70. (2000).

49. Ouyang, Z., Blevins, J. S. \& Norgard, M. V. Transcriptional interplay among the regulators Rrp2, RpoN and RpoS in Borrelia burgdorferi. Microbiology (Reading) 154, 2641-2658 (2008). 
1

2

3

4

5

6

7

8

9

10

11

12

13

14

15

16

17

50. Narasimhan, S. et al. Borrelia burgdorferi transcriptome in the central nervous system of non-human primates. Proc. Natl. Acad. Sci. U.S.A. 100, 15953-15958 (2003).

51. Counter, C. M. et al. Telomerase activity is restored in human cells by ectopic expression of hTERT (hEST2), the catalytic subunit of telomerase. Oncogene 16, 1217-1222 (1998).

52. Poh, M. et al. Blood vessels engineered from human cells. Lancet 365, 2122-2124 (2005).

53. Schneider, C. A., Rasband, W. S. \& Eliceiri, K. W. NIH Image to ImageJ: 25 years of image analysis. Nat. Methods 9, 671-675 (2012).

54. Edelstein, A., Amodaj, N., Hoover, K., Vale, R. \& Stuurman, N. Computer control of microscopes using $\mu$ Manager. Curr Protoc Mol Biol Chapter 14, Unit14.20 (2010).

55. Betteridge, K. B. et al. Sialic acids regulate microvessel permeability, revealed by novel in vivo studies of endothelial glycocalyx structure and function. J. Physiol. (Lond.) 595, 50155035 (2017).

56. Javid, A. et al. Hyperglycemia impairs neutrophil-mediated bacterial clearance in mice infected with the Lyme disease pathogen. PLoS ONE 11, e0158019 (2016).

57. Tang, T. T., Zhang, L., Bansal, A., Grynpas, M. \& Moriarty, T. J. The Lyme disease pathogen Borrelia burgdorferi infects murine bone and induces trabecular bone loss. Infect. Immun. 85, e00781-16 (2017). 


\section{SUPPLEMENTAL TABLES}

Table S1: Correlation analysis: tissue burden in vivo vs flow chamber interaction parameters, $1 \mathrm{~h}, 1 \mathrm{~d}, 1 \mathrm{w}$ post-IV inoculation

\begin{tabular}{|c|c|c|c|c|}
\hline Parameter $^{a}$ & $\begin{array}{c}\text { Bladder } \\
(r, p)^{b}\end{array}$ & $\begin{array}{c}\text { Brain } \\
(r, p)\end{array}$ & $\begin{array}{l}\text { Joint } \\
(r, p)\end{array}$ & $\begin{array}{c}\text { HUVEC/Skin } \\
(r, p)\end{array}$ \\
\hline \multicolumn{5}{|c|}{ Interaction Velocity <175: ALL } \\
\hline Velocity $(\mu \mathrm{m} / \mathrm{sec})$ & $<0.05,>0.95$ & $0.50,0.67$ & $0.50,0.67$ & $>0.95,<0.05$ \\
\hline$K_{\text {off }}\left(s^{-1}\right)$ & $0.50,0.67$ & $0.50,0.67$ & $<0.05,>0.95$ & $<0.05,>0.95$ \\
\hline Displacement $(\mu \mathrm{m})$ & $>-0.95,<0.05$ & $0.50,0.67$ & $0.50,0.67$ & $<0.05,>0.95$ \\
\hline$F_{b}(p N)$ & $<0.05,>0.95$ & $<0.05,>0.95$ & $<0.05,>0.95$ & $<0.05,>0.95$ \\
\hline Interactions/track & $>0.95,<0.05$ & $<0.05,>0.95$ & $<0.05,>0.95$ & $<0.05,>0.95$ \\
\hline \multicolumn{5}{|l|}{ Velocity <175: $-T$} \\
\hline Velocity $(\mu \mathrm{m} / \mathrm{sec})$ & $0.50,0.67$ & $0.50,0.67$ & $<0.05,>0.95$ & $<0.05,>0.95$ \\
\hline$K_{\text {off }}\left(s^{-1}\right)$ & $0.50,0.67$ & $0.50,0.67$ & $<0.05,>0.95$ & $-0.50,0.67$ \\
\hline Displacement $(\mu \mathrm{m})$ & $<0.05,>0.95$ & $0.50,0.67$ & $<0.05,>0.95$ & $<0.05,>0.95$ \\
\hline$F_{b}(p N)$ & $<0.05,>0.95$ & $<0.05,>0.95$ & $<0.05,>0.95$ & $<0.05,>0.95$ \\
\hline Interactions/track & $>0.95,<0.05$ & $0.50,0.67$ & $<0.05,>0.95$ & $<0.05,>0.95$ \\
\hline \multicolumn{5}{|l|}{ Velocity <175: $+T$} \\
\hline Velocity $(\mu \mathrm{m} / \mathrm{sec})$ & $<0.05,>0.95$ & $0.50,0.67$ & $0.50,0.67$ & $<0.05,>0.95$ \\
\hline$K_{o f f}\left(s^{-1}\right)$ & $<0.05,>0.95$ & $<0.05,>0.95$ & $<0.05,>0.95$ & $<0.05,>0.95$ \\
\hline Displacement $(\mu \mathrm{m})$ & $>0.95,<0.05$ & $>0.95,<0.05$ & $0.50,0.67$ & $<0.05,>0.95$ \\
\hline$\left.F_{b}(p N)\right)$ & $<0.05,>0.95$ & $0.50,0.67$ & $<0.05,>0.95$ & $>0.95,<0.05$ \\
\hline Interactions/track & $0.50,0.67$ & $0.50,0.67$ & $<0.05,>0.95$ & $<0.05,>0.95$ \\
\hline
\end{tabular}

a interaction parameters calculated from particle tracking data, organized by interaction type: all, $-T$ (untethered), $+T$ (tethered).

${ }^{b}$ Pearson correlation coefficients, $r$ (strain fold differences in tissue-specific burden in vivo vs strain fold differences in EC-

specific flow chamber interaction numbers) and correlation p-values are indicated above each interaction group.

Table S2: Primary human endothelial cell types used in this study

\begin{tabular}{|l|c|c|c|c|c|c|}
\hline Vascular bed & Cell Type & Abbreviation & $\begin{array}{c}\text { Single or multiple } \\
\text { donors }\end{array}$ & Sex & Source & $\begin{array}{c}\text { Catalog } \\
\text { number }\end{array}$ \\
\hline Aorta & arterial & HAEC & single & female & Lonza Inc & CC-2535 \\
\hline Bladder & microvascular & HMVEC- Bd & single & female & Lonza Inc & CC-7016 \\
\hline Brain cortex & microvascular & HMVEC-Br & single & male & Cell Systems & ACBRI 376 \\
\hline Synovial tissue & microvascular & HMVEC-Joint & single & female & Cell Systems & ACBRI 488 \\
\hline Umbilical cord vein & venous & HUVEC & multiple & female & Lonza Inc & CC-2519 \\
\hline
\end{tabular}

a all endothelia were derived from healthy donors.

Table S3: B. burgdorferi strains used in this study

\begin{tabular}{|c|c|c|c|c|c|}
\hline Strain & Background & Description & $\begin{array}{c}\text { Antibiotic } \\
\text { resistance }^{a}\end{array}$ & $\begin{array}{c}\text { No flow } \\
\text { bacterial } \\
\text { length }(\mu m)^{b}\end{array}$ & References \\
\hline \begin{tabular}{|l|} 
GCB726 \\
$(+1 \mathrm{lp} 25$ \\
$-b b k 32)$ \\
\end{tabular} & B31 5A4 NP1 & $\begin{array}{c}\text { infectious B31 5A4 NP1 derivative expressing } \\
P_{f l a B} \text {-driven GFP; contains all endogenous } \\
\text { plasmids except cp9 }\end{array}$ & $\begin{array}{l}\text { gentamicin, } \\
\text { kanamycin }\end{array}$ & 15.40 & 11 \\
\hline \begin{tabular}{|c|} 
GCB966 \\
$(-\operatorname{lp} 25$ \\
$+b b k 32)$ \\
\end{tabular} & $\begin{array}{l}\text { B31 ML23 derivative } \\
\text { (ML23/pJW201) }\end{array}$ & $\begin{array}{c}\text { infectious ML23-derived strain expressing } P_{f l a B} \\
\text {-driven GFP; lacks lp25; bbe22 locus carried on } \\
\text { plasmid pJW201 }\left(P_{f l a b}-\mathrm{GFP} / \mathrm{bbe} 22\right)\end{array}$ & gentamicin & 14.85 & 10 \\
\hline \begin{tabular}{|c|} 
GCB971 \\
$(-1 \mathrm{p} 25$ \\
$-b b k 32)$ \\
\end{tabular} & $\begin{array}{l}\text { B31 ML23 derivative } \\
\text { (JS315/pJW201) }\end{array}$ & $\begin{array}{l}\text { ML23-derived } b b k 32: s t r R \text { strain expressing } P_{f l a B} \\
\text {-driven GFP; isogenic parent: GCB9666 }\end{array}$ & $\begin{array}{l}\text { gentamicin, } \\
\text { streptomycin }\end{array}$ & 13.31 & 10 \\
\hline
\end{tabular}

${ }^{a}$ Antibiotic concentrations: gentamicin (100 $\left.\mu \mathrm{g} / \mathrm{ml}\right)$, kanamycin $(200 \mu \mathrm{g} / \mathrm{ml})$, streptomycin $\left(50 \mu \mathrm{g} / \mathrm{ml} .{ }^{b}\right.$ This study. Average length of single (unreplicated) bacteria for indicated strains, measured by life cell imaging in flow chambers and particle tracking of time lapses acquired using the Leica microscope under no flow conditions. 
Table S4: Particle tracking conditions

\begin{tabular}{|c|c|c|c|c|}
\hline Protocol & Parameters & GCB726 & GCB966 & GCB971 \\
\hline \multirow[t]{5}{*}{ A } & Object size $\left(\mu \mathrm{m}^{2}\right): 1.5-1.1 \mathrm{X}$ no-flow bacterial length $(\mathrm{BL})$ & $1.5-16.95$ & $1.5-16.33$ & $1.5-14.64$ \\
\hline & Object Skeletal length $(\mu \mathrm{m}): 2.5-1 \mathrm{X}$ BL & $2.5-15.4$ & $2.5-14.85$ & $2.5-13.31$ \\
\hline & Object Intensity & \multicolumn{3}{|c|}{$17-255$} \\
\hline & Object joining (tracking) algorithm & \multicolumn{3}{|c|}{$\begin{array}{c}\text { Trajectory Variation (0.01), Estimate Max Distance } \\
\text { between Objects Automatically }\end{array}$} \\
\hline & Maximum Track Velocity $(\mu \mathrm{m} / \mathrm{s})$ & \multicolumn{3}{|c|}{300} \\
\hline \multirow[t]{5}{*}{$\mathrm{B}$} & Object size $\left(\mu \mathrm{m}^{2}\right): 1.5-1.1 \mathrm{X}$ no-flow bacterial length (BL) & $1.2-53.28$ & $1.2-51.38$ & $1.2-46.05$ \\
\hline & Object Skeletal length $(\mu \mathrm{m}): 2.5-1 \mathrm{X} B \mathrm{~B}$ & $1.4-15.4$ & $1.4-14.85$ & $1.4-13.31$ \\
\hline & Object Intensity & \multicolumn{3}{|c|}{$40-135$} \\
\hline & Object joining (tracking) algorithm & \multicolumn{3}{|c|}{ See details above in protocol A } \\
\hline & Maximum Track Velocity $(\mu \mathrm{m} / \mathrm{s})$ & \multicolumn{3}{|c|}{$\leq 185 \mu \mathrm{m} / \mathrm{sec}$} \\
\hline \multirow[t]{5}{*}{$\mathrm{C}$} & Object size $\left(\mu \mathrm{m}^{2}\right): 1.5-1.1 \mathrm{X}$ no-flow bacterial length (BL) & $5.0-53.28$ & $5.0-51.38$ & $5.0-46.05$ \\
\hline & Object Skeletal length $(\mu \mathrm{m}): 2.5-1 \mathrm{X}$ BL & $1.4-15.4$ & $1.4-14.85$ & $1.4-13.31$ \\
\hline & Object Intensity & \multicolumn{3}{|c|}{$40-135$} \\
\hline & Object joining (tracking) algorithm & \multicolumn{3}{|c|}{ See details above in protocol A } \\
\hline & Maximum Track Velocity $(\mu \mathrm{m} / \mathrm{s})$ & \multicolumn{3}{|c|}{$\leq 300 \mu \mathrm{m} / \mathrm{sec}$} \\
\hline \multirow[t]{5}{*}{$\mathrm{D}$} & Object size $\left(\mu \mathrm{m}^{2}\right): 1.5-1.1 \mathrm{X}$ no-flow bacterial length (BL) & $1.2-53.28$ & $1.2-51.38$ & $1.2-46.05$ \\
\hline & Object Skeletal length $(\mu \mathrm{m}): 2.5-1 \mathrm{X}$ BL & $1.4-15.4$ & $1.4-14.85$ & $1.4-13.31$ \\
\hline & Object Intensity & \multicolumn{3}{|c|}{$110-255$} \\
\hline & Object joining (tracking) algorithm & \multicolumn{3}{|c|}{ See details above in protocol A } \\
\hline & Maximum Track Velocity $(\mu \mathrm{m} / \mathrm{s})$ & \multicolumn{3}{|c|}{$\leq 185 \mu \mathrm{m} / \mathrm{sec}$} \\
\hline \multirow[t]{5}{*}{$\mathrm{E}$} & Object size $\left(\mu \mathrm{m}^{2}\right): 1.5-1.1 \mathrm{X}$ no-flow bacterial length (BL) & $5.0-53.28$ & $5.0-51.38$ & $5.0-46.05$ \\
\hline & Object Skeletal length $(\mu \mathrm{m}): 2.5-1 \mathrm{X}$ BL & $1.4-15.4$ & $1.4-14.85$ & $1.4-13.31$ \\
\hline & Object Intensity & \multicolumn{3}{|c|}{$110-255$} \\
\hline & Object joining (tracking) algorithm & \multicolumn{3}{|c|}{ See details above in protocol A } \\
\hline & Maximum Track Velocity $(\mu \mathrm{m} / \mathrm{s})$ & \multicolumn{3}{|c|}{$\leq 300 \mu \mathrm{m} / \mathrm{sec}$} \\
\hline \multirow[t]{5}{*}{$\mathrm{F}$} & Object size $\left(\mu \mathrm{m}^{2}\right): 1.5-1.1 \mathrm{X}$ no-flow bacterial length (BL) & $1.5-16.95$ & $1.5-16.33$ & $1.5-14.64$ \\
\hline & Object Skeletal length $(\mu \mathrm{m}): 1.4-1 \mathrm{X}$ BL & $1.4-15.4$ & $1.4-14.85$ & $1.4-13.31$ \\
\hline & Object Intensity & \multicolumn{3}{|c|}{$17-255$} \\
\hline & Object joining (tracking) algorithm & \multicolumn{3}{|c|}{ Shortest Path (Max Distance $15 \mu \mathrm{m})$} \\
\hline & Maximum Track Velocity $(\mu \mathrm{m} / \mathrm{s})$ & \multicolumn{3}{|c|}{200} \\
\hline
\end{tabular}




\section{SUPPLEMENTAL FIGURES}
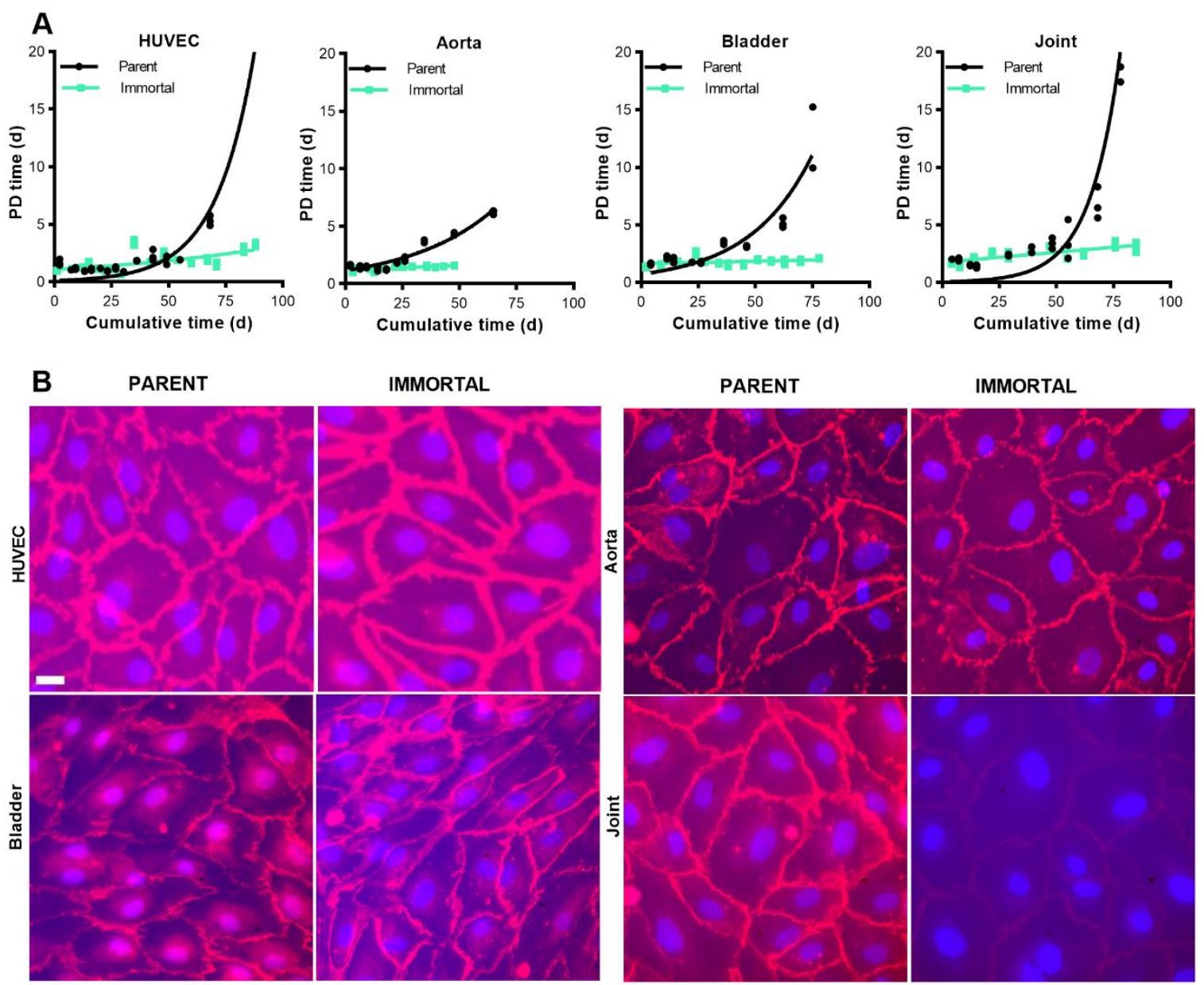

C
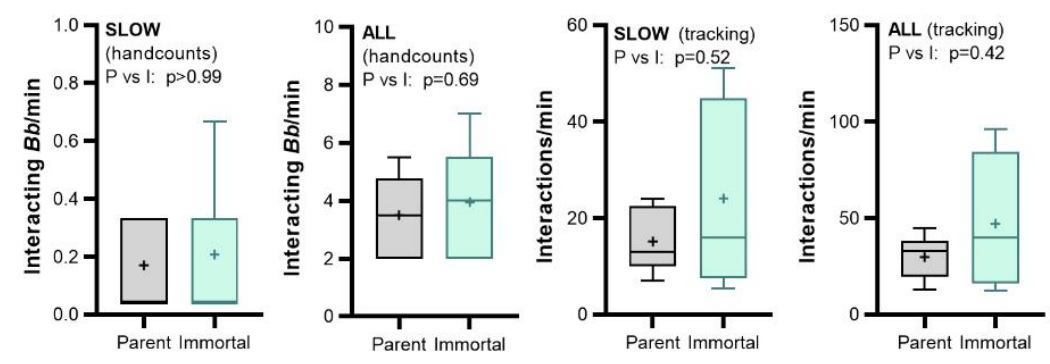

Fig S1: Population doubling times and VE-cadherin expression in parent and immortalized ECs. (A) Population doubling (PD) time plots and non-linear regressions for parent (P) and hTERT-immortalized (I) ECs. (B) IF visualization of VE-cadherin (red) in P and I ECs counterstained with DAPI (blue). Scale bar: $30 \mu \mathrm{m}$. (C) Data are shown as Tukey box and whiskers plots of total (all) and slow (velocity $<125 \mu \mathrm{m} / \mathrm{s}$ ) interaction numbers under flow for all bacterial strains (+lp25+bbk32, -lp25+bbk32, -lp25-bbk32) and ECs derived from aorta, bladder and joint, measured by manual and particle tracking enumeration (hand counts, tracking, respectively). For all Fig. S1 experiments, $\mathrm{N} \geq 3$ independent EC and bacterial cultures. Statistics: Mann-Whitney or Welch's t-test (depending on normality of distribution, determined by normality tests) of log-transformed data. 

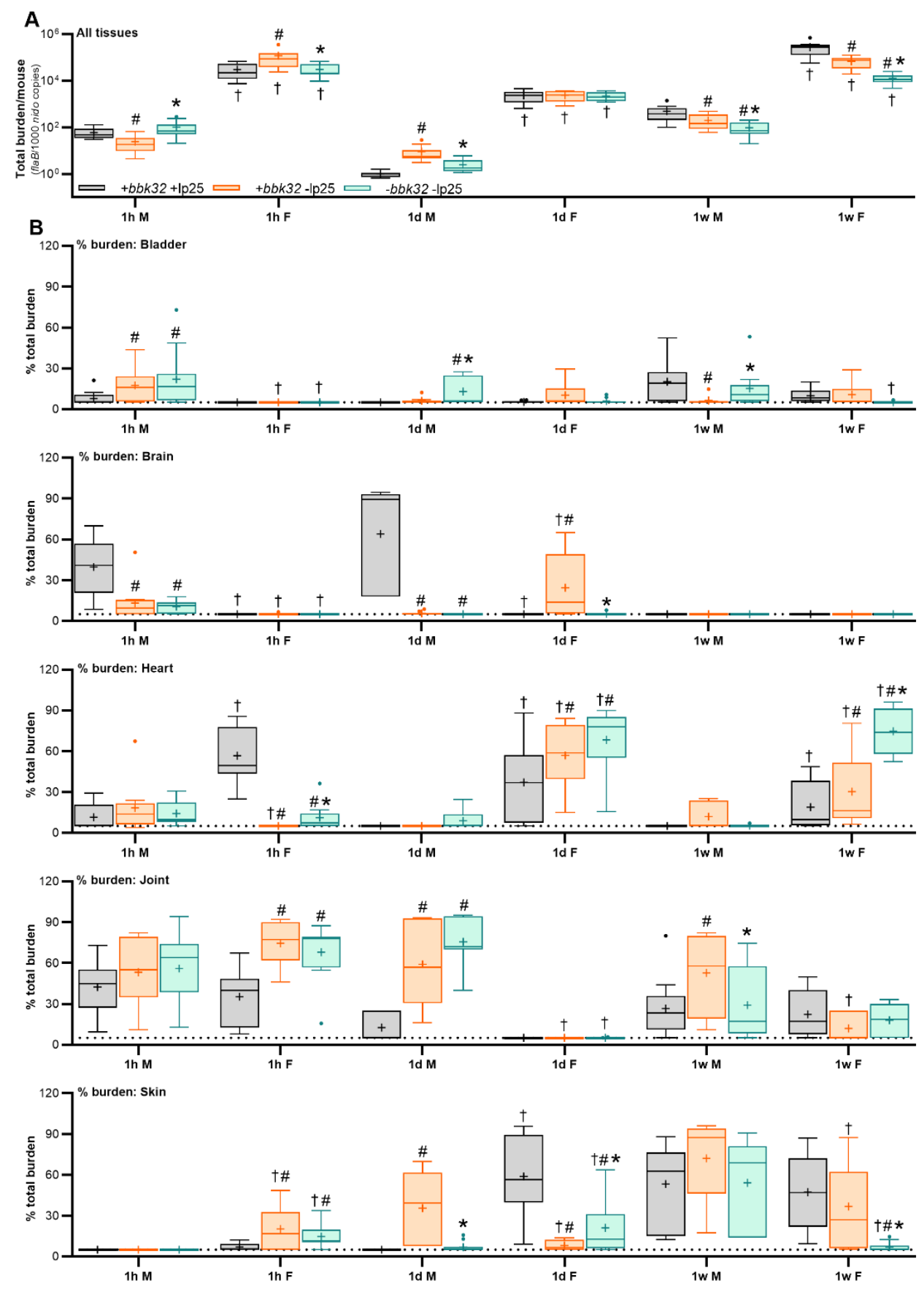

Fig. S2: B. burgdorferi dissemination to tissues after intravenous inoculation of male or female mice. Total $B$. burgdorferi burden in all tissues (A) and percent of total burden in indicated tissues at $1 \mathrm{~h}, 1$ day (d) and 1 week (w) after intravenous inoculation of female and male mice (B), measured by quantitative PCR. Data are shown as Tukey box and whiskers plots. $\mathrm{N} \geq 26$ mice/experimental group. Statistics: two-way ANOVA of log-transformed values, with Holm-Sidak post-tests. * indicates $\mathrm{p}<0.05$ vs $-1 \mathrm{p} 25-b b k 32$. \# indicates $\mathrm{p}<0.05 \mathrm{vs}+\mathrm{lp} 25+b b k 32$. 

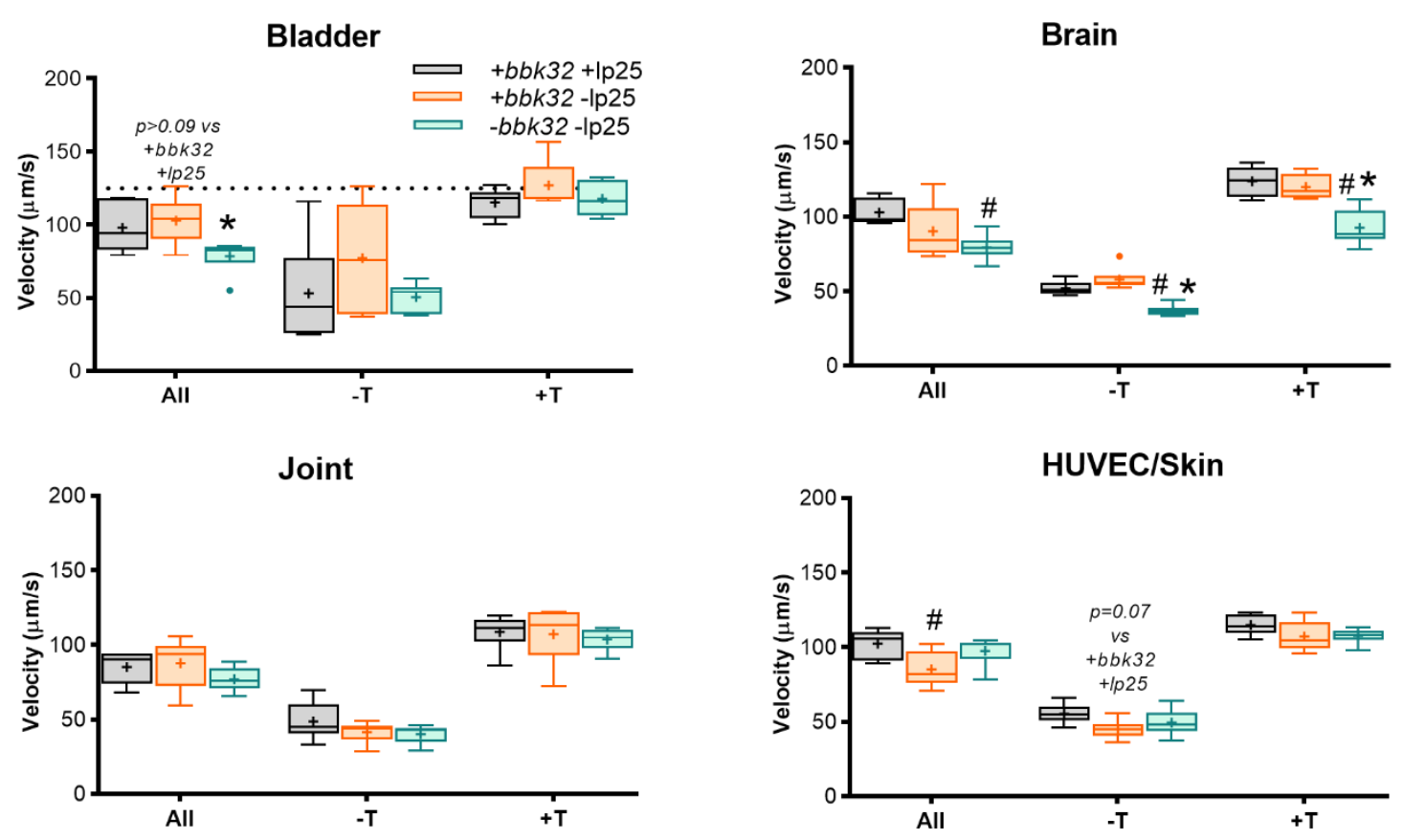

Fig. S3: Bacterial-endothelial interaction velocity in flow chambers predict dissemination patterns in vivo. $B$. burgdorferi-EC interaction velocity $(\mu \mathrm{m} / \mathrm{s}$ :) for all, $-\mathrm{T}$ (untethered) and $+\mathrm{T}$ (tethered) interactions in flow chambers, measured by particle tracking. Data shown as Tukey box and whiskers plots. $\mathrm{N} \geq 3$ biological replicates/experimental group. Statistical comparisons within each interaction group: ANOVA with Tukey's post-tests. $*$ indicates $\mathrm{p}<0.05$ vs lp25+bbk32. \# indicates $\mathrm{p}<0.05$ vs $+\mathrm{lp} 25+b b k 32$. Pearson correlation coefficients, $r$ (strain fold differences in tissuespecific burden in vivo vs strain fold differences in EC-specific flow chamber interaction numbers) and correlation $p$ values are indicated above each interaction group. Correlation values for all other interaction parameters are provided in Tables 1 and S1.

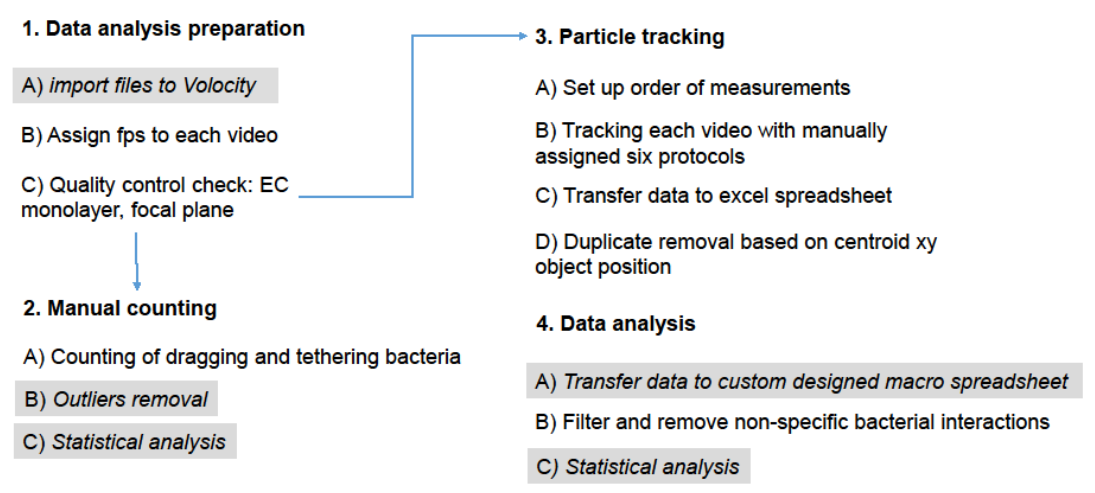

Fig. S4: Bioinformatics summary of particle tracking and measuring biophysical properties of $B$. burgdorferiendothelial interactions under flow. Quality control and analysis steps in the time lapse bioinformatics. Highlighted in grey are steps performed using automated spreadsheets and software (Volocity, GraphPad Prism). Other steps are performed manually. 